Network Working Group

Request for Comments: 4149

Category: Standards Track
C. Kalbfleisch

Consultant

R. Cole

$\mathrm{JHU} / \mathrm{APL}$

D. Romascanu

Avaya

August 2005

\title{
Definition of Managed Objects for Synthetic Sources for Performance Monitoring Algorithms
}

Status of This Memo

This document specifies an Internet standards track protocol for the Internet community, and requests discussion and suggestions for improvements. Please refer to the current edition of the "Internet Official Protocol Standards" (STD 1) for the standardization state and status of this protocol. Distribution of this memo is unlimited.

Copyright Notice

Copyright (C) The Internet Society (2005) .

Abstract

This memo defines a portion of the Management Information Base (MIB) for use with network management protocols in the Internet community. In particular, it describes objects for configuring synthetic sources for Performance Monitoring (SSPM) algorithms. 
Table of Contents

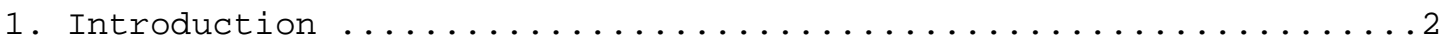

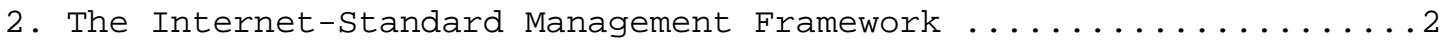

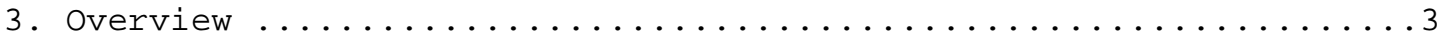

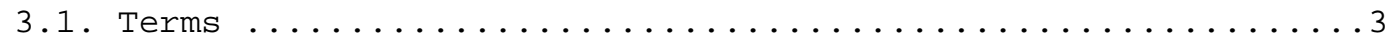

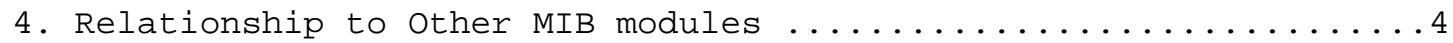

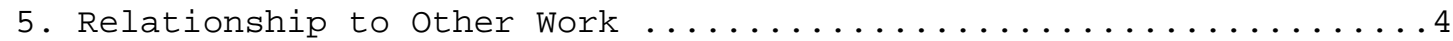

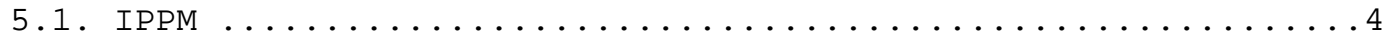

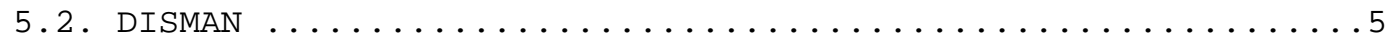

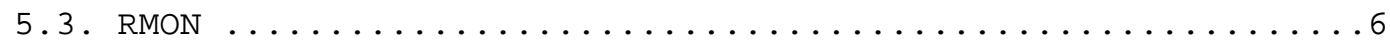

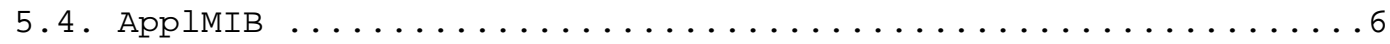

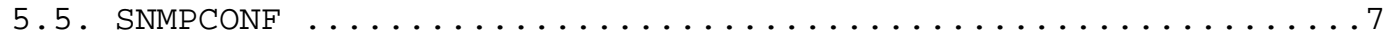

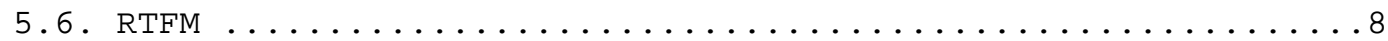

5.7. Relationship to Other Work: Summary ...............

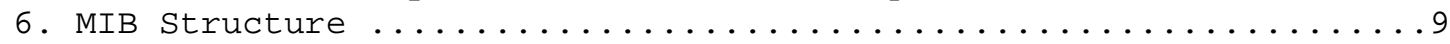

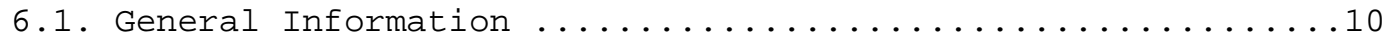

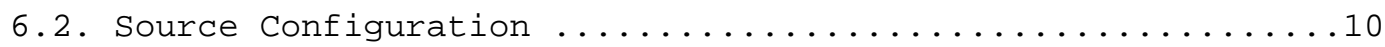

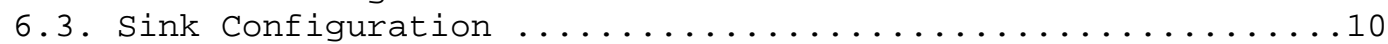

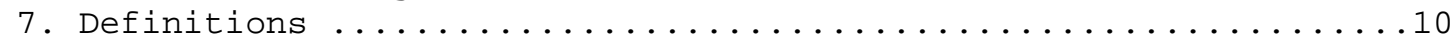

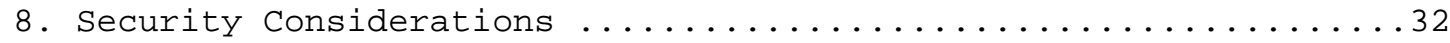

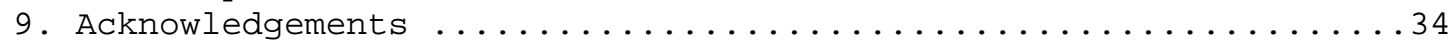

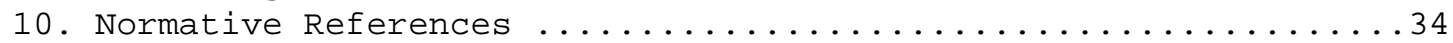

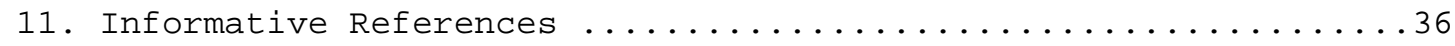

\section{Introduction}

This memo defines a portion of the Management Information Base (MIB) for use with network management protocols in the Internet community.

In particular, it defines a method of describing synthetic sources for Performance Monitoring (SSPM). This is useful within the Remote Monitoring (RMON) framework [RFC3577] for performance monitoring in the cases where it is desirable to inject packets into the network for the purpose of monitoring their performance with the other MIBs in that framework.

This memo also includes a MIB module.

The key words "MUST", "MUST NOT", "REQUIRED", "SHALL", "SHALL NOT", "SHOULD", "SHOULD NOT", "RECOMMENDED", "MAY", and "OPTIONAL" in this document are to be interpreted as described in RFC 2119 [RFC2119].

2. The Internet-Standard Management Framework

For a detailed overview of the documents that describe the current Internet-Standard Management Framework, please refer to section 7 of RFC 3410 [RFC3410]. 
Managed objects are accessed via a virtual information store, termed the Management Information Base or MIB. MIB objects are generally accessed through the Simple Network Management Protocol (SNMP). Objects in the MIB are defined using the mechanisms defined in the Structure of Management Information (SMI). This memo specifies a MIB module that is compliant to the SMIV2, which is described in STD 58, RFC 2578 [RFC2578], STD 58, RFC 2579 [RFC2579] and STD 58, RFC 2580 [RFC2580].

3. Overview

This document defines a MIB module for the purpose of remotely controlling synthetic sources (or 'active' probes) and sinks in order to enhance remote performance monitoring capabilities within IP networks and services. Much work within the IETF exists related to performance monitoring. One interesting aspect of this body of work is that it does not explicitly define an 'active' probe capability. An active probe capability is complimentary to existing capabilities, and this MIB module is developed to fill this void.

\subsection{Terms}

The following definitions apply throughout this document:

o 'Performance monitoring' is the act of monitoring traffic for the purpose of evaluating a statistic of a metric related to the performance of the system. A performance monitoring system is comprised of a) traffic generators, b) measurement, c) data reduction, and d) reporting. The traffic generators may be natural sources, synthetic sources, or intrusive sources.

o A 'synthetic source' is a device or an embedded software program that generates a data packet (or packets) and injects it (or them) onto the path to a corresponding probe or existing server solely in support of a performance monitoring function. A synthetic source may talk intrusively to existing application servers.

The design goals for this MIB module are:

- Complementing the overall performance management architecture being defined within the RMONMIB WG; refer to the RMONMIB framework document [RFC3577]. This MIB module is defined within the context of the APM-MIB [RFC3729].

- Extensibility: the MIB module should be easily extended to include a greater set of protocols and applications for performance monitoring purposes. 
o Flexibility: the module should support both round-trip and oneway measurements.

o Security: the control of the source and sink of traffic is handled by a management application, and communication is recommended via SNMPV3.

This document is organized as follows. The next section discusses the relationship of this MIB module to others from the RMONMIB and Distributed Management (DISMAN) working groups. Then the structure of the MIB module is discussed. Finally, the MIB module definitions are given.

4. Relationship to Other MIB modules

This MIB module is designed to be used in conjunction with the RMON MIB Working Group's two other MIB modules for application performance measurement: Application Performance Measurement MIB [RFC3729] and Transport Performance Metrics MIB [RFC4150]. These MIB modules define reporting capabilities for that framework. The intent of this MIB module is to define a method for injecting packets into the network utilizing probe capabilities defined in the base MIB modules and measured with the reporting MIB modules. Other reporting MIB modules may be used as well.

Specifically, this MIB module uses the AppLocalIndex as defined in the APM-MIB to map measurement configuration information to definition and reporting structures defined in the APM-MIB.

5. Relationship to Other Work

Much work has already been done within the IETF that has a direct bearing on the development of active performance probe definitions. This body of work has been addressed in various working groups over the years. In this section, we focus on the work of a) the IP Performance Metrics (IPPM) working group, b) the DISMAN working group, c) the RMON working group, d) the Application MIB (ApplMIB) working group, and e) the Realtime Traffic Flow Measurement (RTFM) working group.

5.1. IPPM

The IPPM working group has defined in detail a set of performance metrics, sampling techniques, and associated statistics for transport-level or connectivity-level measurements. The IPPM framework document [RFC2330] discusses numerous issues concerning sampling techniques, clock accuracy, resolution and skew, wire time versus host time, error analysis, etc. Many of these are 


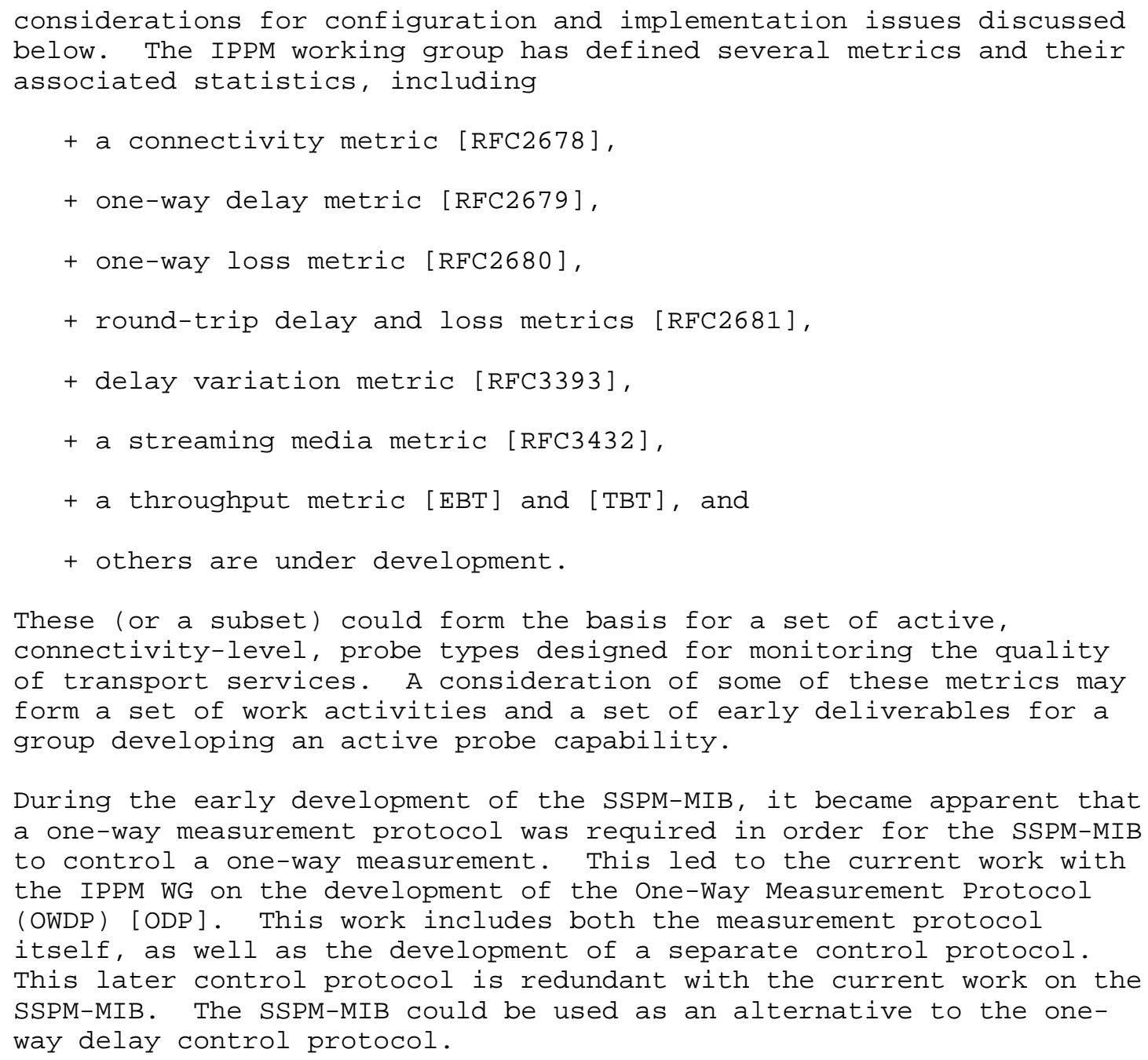

The DISMAN working group has defined a set of 'active' tools for remote management. Of relevance to this document are:

+ the pingMIB [RFC2925],

+ the DNS Lookup MIB [RFC2925],

+ the tracerouteMIB [RFC2925], 
+ the scriptMIB [RFC3165], and

+ the expressionMIB [RFC2982].

The pingMIB and tracerouteMIB define an active probe capability, primarily for the remote determination of path and path connectivity. There are some performance-related metrics collected from the pingMIB, and one could conceivably use these measurements for the evaluation of a limited set of performance statistics. But there is a fundamental difference between determining connectivity and determining the quality of that connectivity. However, in the context of performance monitoring, a fault can be viewed as not performing at all. Therefore, both should be monitored with the same probes to reduce network traffic.

The DNS Lookup MIB also includes some probe-like capabilities and performance time measurements for the DNS lookup. This could be used to suggest details of a related session-level, active probe.

The scriptMIB allows a network management application to distribute and manage scripts to remote devices. Conceivably, these scripts could be designed to run a set of active probe monitors on remote devices.

\subsection{RMON}

The RMON working group has developed an extensive, passive monitoring capability defined in RFC 2819 [RFC2819] and RFC 2021 [RFC2021] as well as additional MIB modules. Initially, the monitors collected statistics at the MAC layer, but the capability has now been extended to higher-layer statistics. Higher-layer statistics are identified through the definition of a Protocol Directory [RFC2021]. See the RMONMIB framework document [RFC3577] for an overview of the RMONMIB capabilities.

Within this context, the development of an active traffic source for performance monitoring fits well within the overall performance monitoring architecture being defined within the RMON WG.

\section{4. ApplMIB}

The ApplMIB working group defined a series of MIB modules that monitor various aspects of applications, processes, and services.

The System Application MIB [RFC2287] describes a basic set of managed objects for fault, configuration, and performance management of applications from a systems perspective. More specifically, the managed objects it defines are restricted to information that can be 
determined from the system itself and that does not require special instrumentation within the applications to make the information available.

The Application MIB [RFC2564] complements the System Application MIB, providing for the management of applications' common attributes, which could not typically be observed without the cooperation of the software being managed. There are attributes that provide information on application and communication performance.

The WWW MIB [RFC2594] describes a set of objects for managing networked services in the Internet Community, particularly World Wide Web (WWW) services. Performance attributes are available for the information about each WWW service, each type of request, each type of response, and top-accessed documents.

In the development of synthetic application-level probes, consideration should be given to the relationship of the application MIB modules to the measurements being performed through a synthetic application-level probe. Similar, cross-indexing issues arise within the context of the RMON monitoring and synthetic application-level active probes.

\section{5. SNMPCONF}

The Configuration Management with SNMP (SNMPCONF) working group has created the informational RFC 3512 [RFC3512], which outlines the most effective methods for using the SNMP Framework to accomplish configuration management. This work includes recommendations for device-specific as well as network-wide (Policy) configuration. The group is also chartered to write any MIB modules necessary to facilitate configuration management. Specifically, they will write a MIB module that describes a network entity's capabilities and capacities, which can be used by management entities making policy decisions at a network level or device-specific level.

Currently, the SNMPCONF working group is focused on the SNMP Configuration MIB for policy [RFC4011]. It is conceivable that one would want to monitor the performance of newly configured policies as they are implemented within networks. This would require correlation of the implemented policy and a related performance monitoring policy that would specify synthetic probe definitions. For synthetic probes, there would be a need for a configuration of a) a single probe, b) several probes, c) source and destination probes, and d) intermediate probes. In addition, it may be necessary to configure any or all of these combinations simultaneously. It is hoped that the work of SNMPCONF will suffice. The scripting language defined by the SNMP Configuration MIB could allow for active monitoring to be 
activated and configured from a policy management script. Further, the results of active monitoring could become arguments in further policy decisions. This notion is reflected in the decision flow outlined in Figure 1 below.

\subsection{RTFM}

The Realtime Traffic Flow Measurement (RTFM) working group is concerned with issues relating to traffic flow measurements and usage reporting for network traffic and Internet accounting. Various documents exist that describe requirements [RFC1272], traffic flow measurement architectures [RFC2722], and a traffic flow MIB [RFC2720]. The work in this group is focused on passive measurements of user traffic. As such, its work is related to the monitoring work within the RMON WG. Fundamentally, their attention has not been concerned with methods of active traffic generation.

\subsection{Relationship to Other Work: Summary}

In summary, the development of an active traffic generation capability (primarily for the purpose of performance monitoring) should draw upon various activities, both past and present, within the IETF. Figure 1 shows the relationship of the various work activities briefly touched upon in this section.

Horizontally, across the top of the figure are overall control functions, which would coordinate the various aspects of the performance monitoring systems. Vertically at the bottom of the figure are the functions which comprise the minimum performance monitoring capability; i.e., traffic generation, monitoring and measurements, and data reduction. Traffic generation is addressed in this MIB module. Monitoring and measurement is addressed in the APM-MIB [RFC3729] and TPM-MIB [RFC4150] modules. Data reduction is not yet addressed within the IETF. But data reduction could include both spatial and temporal aggregations at different levels of reduction. This is indicated in the figure by the arrow labeled "Various levels and span". 


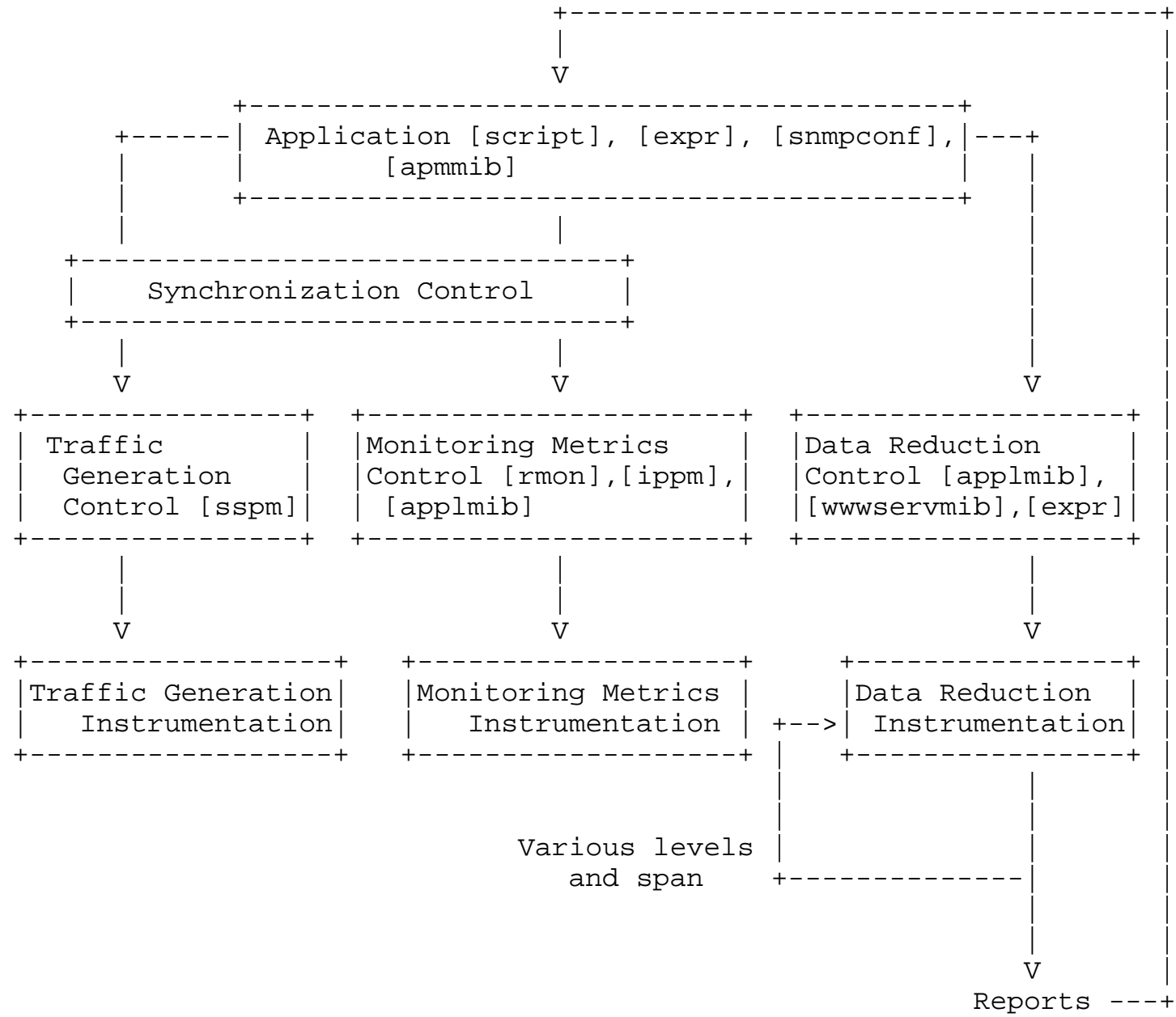

Figure 1: Coverage for an overall performance monitoring system

6. MIB structure

This section presents the structure of the MIB module. The objects are arranged into the following groups:

o general information

o source configuration

o sink configuration 


\subsection{General Information}

This section provides general information about the capabilities of the probe. Currently, this information is related to the resolution of the probe clock and its source.

\subsection{Source Configuration}

The source is configured with a pair of tables. The first, sspmSourceprofileTable, defines a set of profiles for monitoring. These profiles are then used by the second table, sspmsourcecontrolTable, to instantiate a specific measurement. This MIB module takes an IP-centric view of the configuration of the measurement.

\subsection{Sink Configuration}

Configures the sink for measurements. If the test is round-trip, then this table is on the same probe as the source configuration. If the test is one-way, then the table is on a different probe. The sspmSinkInstance is a unique identifier for the entry per probe. Additional attributes are provided for test type and test source to identify entries in the table uniquely.

7. Definitions

SSPM-MIB DEFINITIONS : := BEGIN

IMPORTS

MODULE-IDENTITY, OBJECT-TYPE, Counter32, Integer32, Unsigned32

FROM SNMPV2-SMI -- [RFC2578]

TEXTUAL-CONVENTION, StorageType, Truthvalue, Rowstatus

FROM SNMPV2-TC -- [RFC2579]

MODULE-COMPLIANCE, OBJECT-GROUP

FROM SNMPV2-CONF -- [RFC2578,

-- RFC2579,

- RFC2580]

Ownerstring, rmon

FROM RMON-MIB -- [RFC2819]

InetAddresstype, InetAddress

FROM INET-ADDRESS-MIB -- [RFC3291]

Kalbfleisch, et al.

Standards Track

[Page 10] 


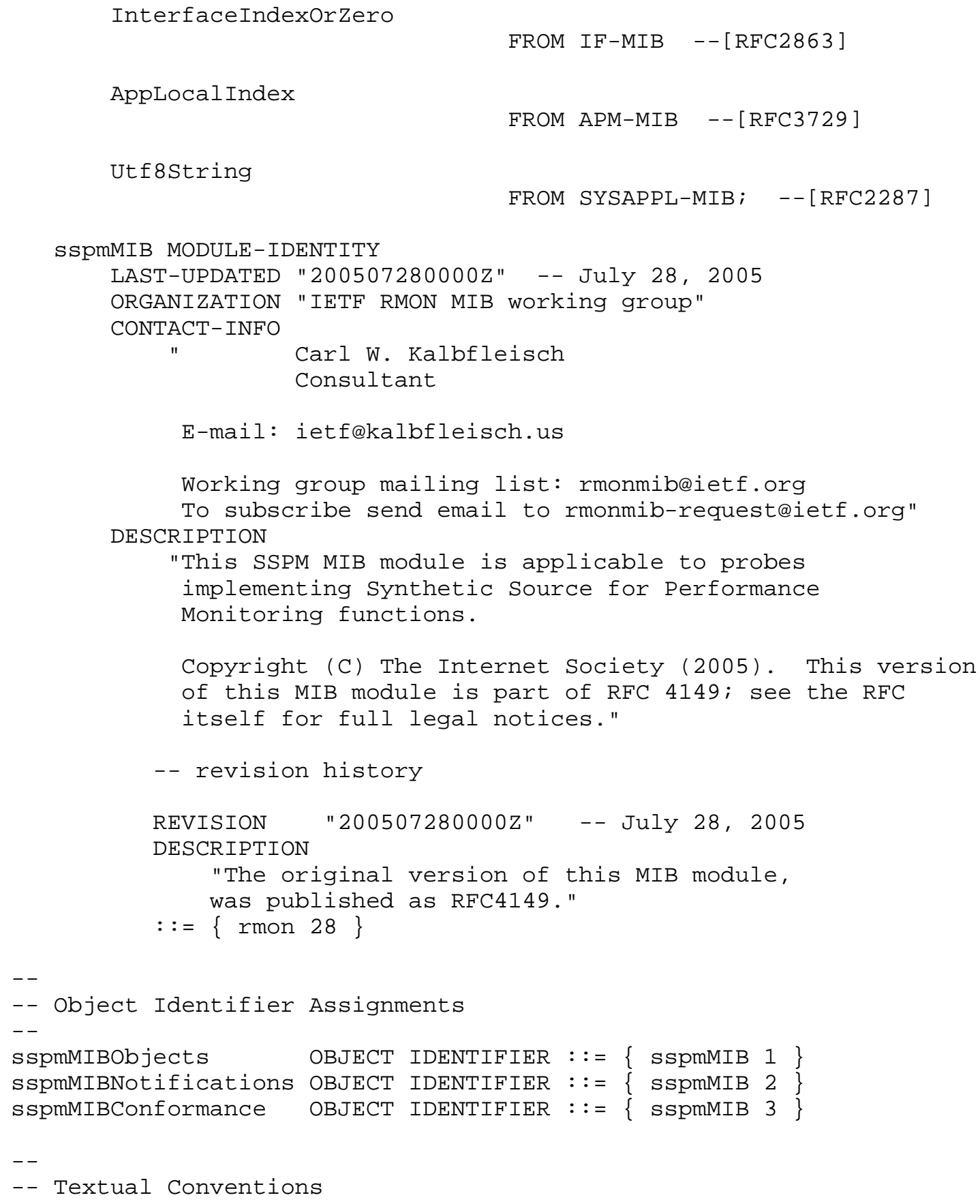




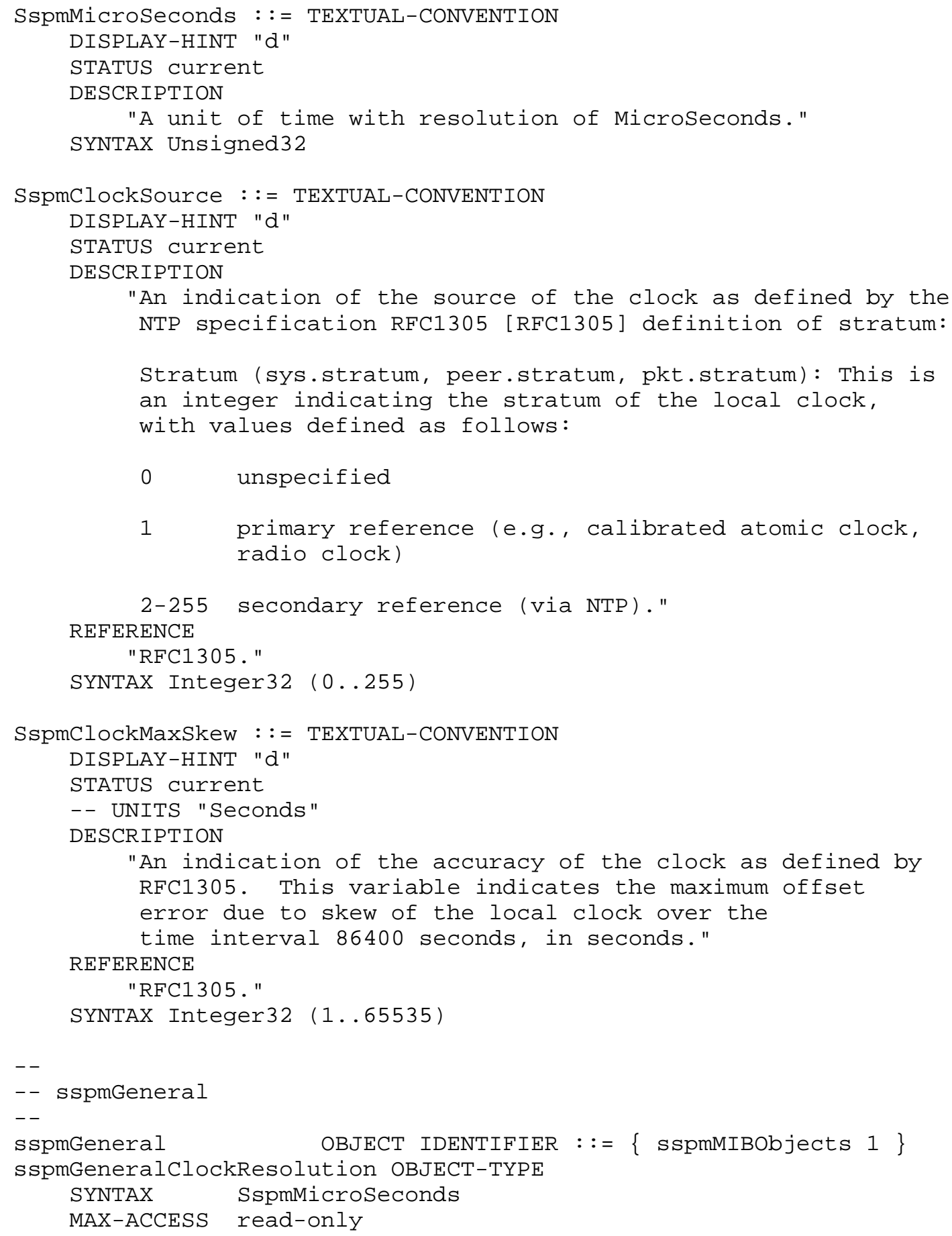




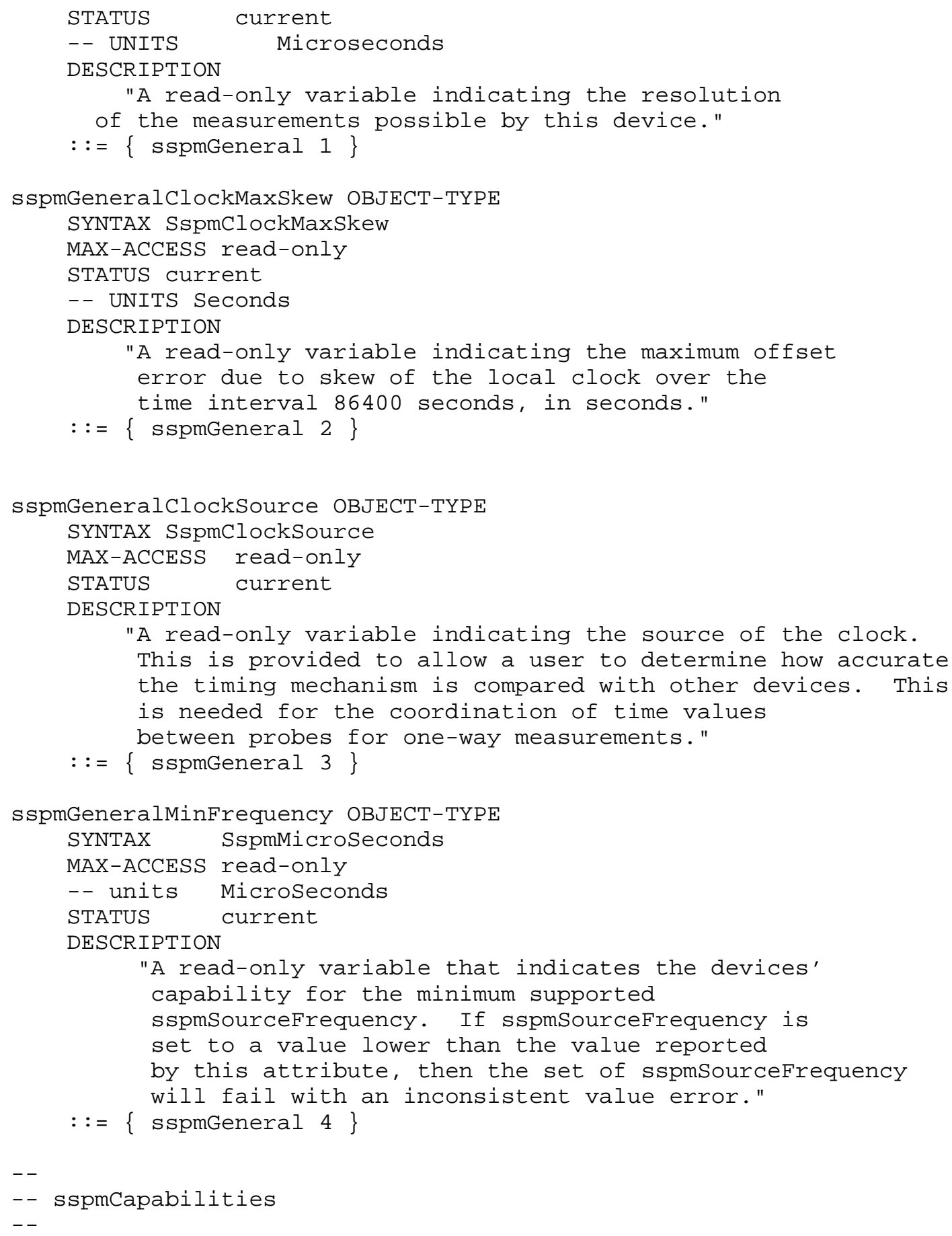




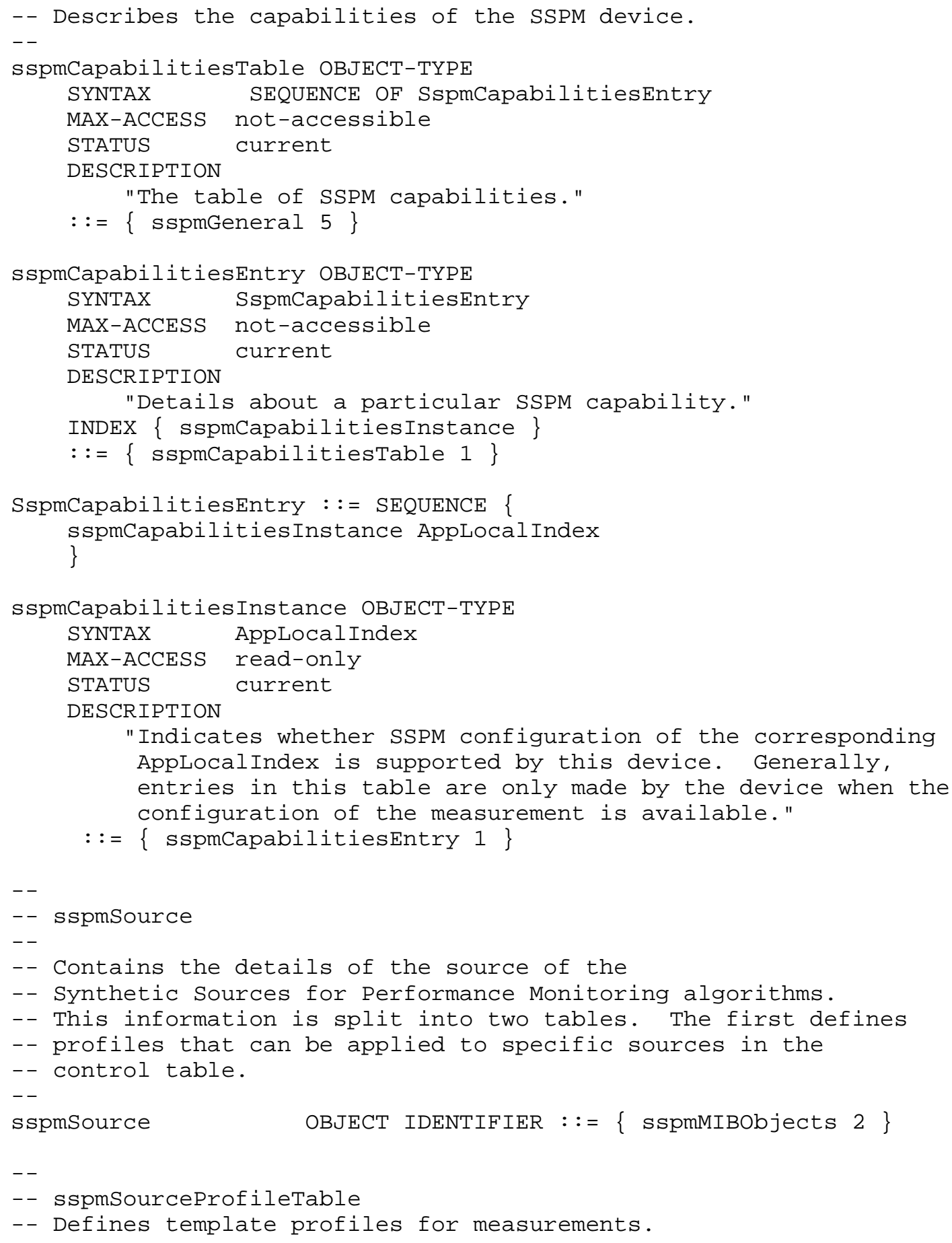




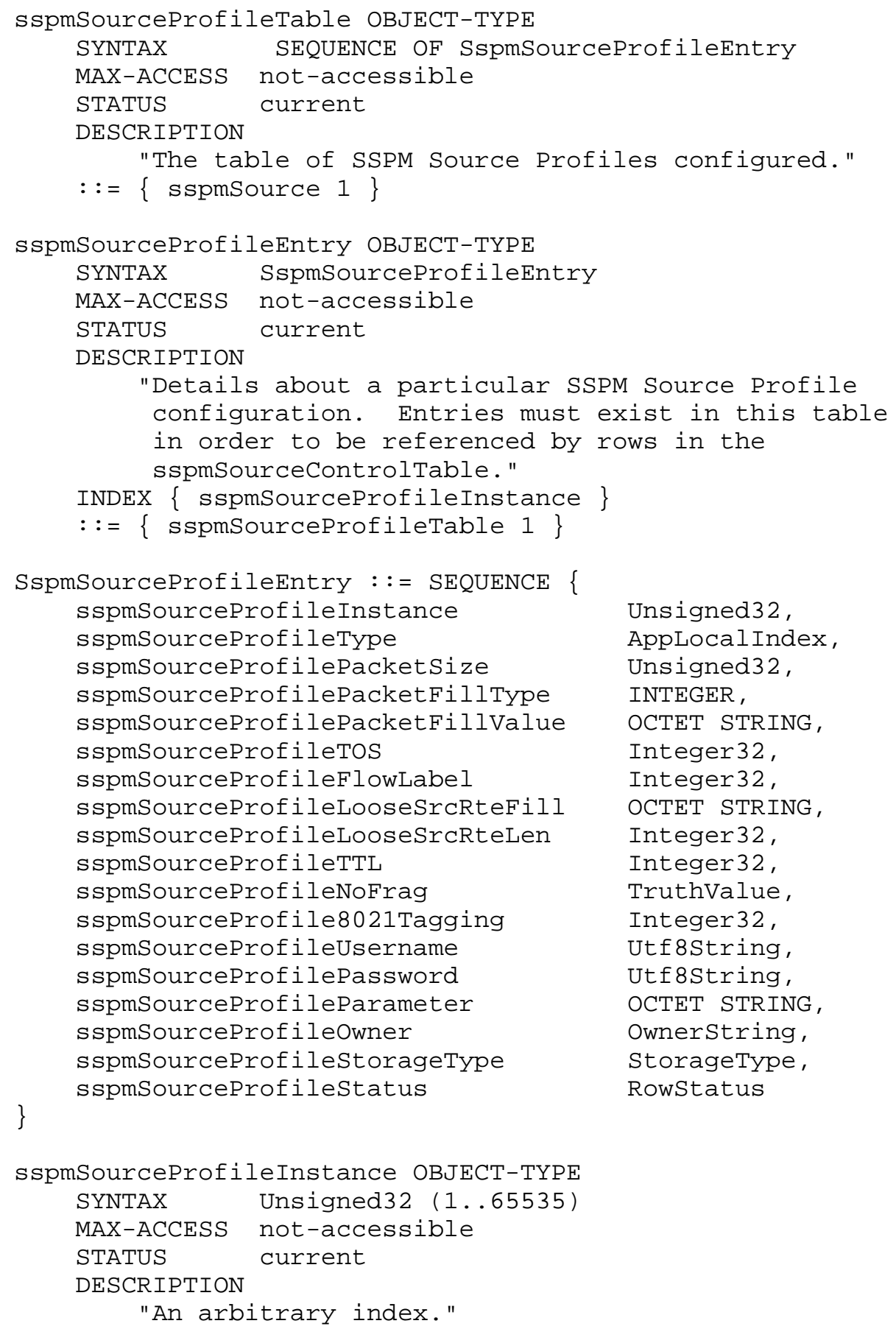


$::=\{$ sspmSourceprofileEntry 1$\}$

sspmSourceProfileType OBJECT-TYPE

SYNTAX AppLocalindex

MAX-ACCESS read-create

STATUS current

DESCRIPTION

"The AppLocalIndex value that uniquely identifies the measurement per the APM-MIB. In order to create a row in this table, there must be a corresponding row in the sspmcapabilitiestable.

When attempting to set this object, if no corresponding row exists in the sspmcapabilitiestable, then the agent should return a 'badValue' error." $::=\{$ sspmSourceProfileEntry 2$\}$

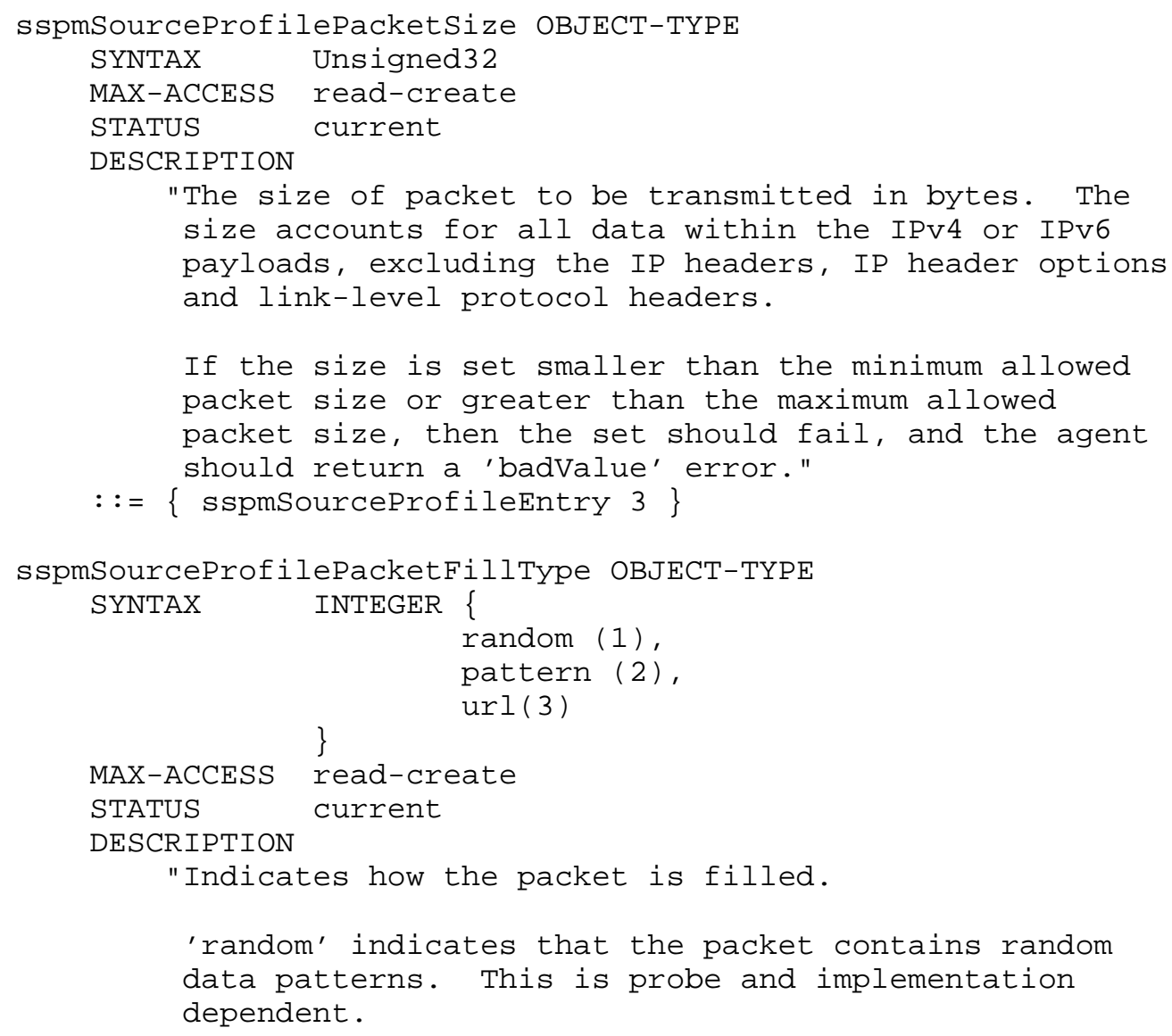




\begin{abstract}
'pattern' indicates that the pattern defined in the sspmSourceProfilePacketFillvalue attribute is used to fill the packet.
\end{abstract}

'url' indicates that the value of sspmSourceProfilePacketFillValue should contain a URL. The contents of the document at that URL are retrieved when sspmsourcestatus becomes active and utilized in the packet. If the attempt to access that URL fails, then the row status is set to 'notReady', and the set should fail with 'inconsistentValue'. This value must contain a dereferencable URL of the type 'http:', 'https:', or 'ftp:' only." $::=\{$ sspmSourceProfileEntry 4$\}$

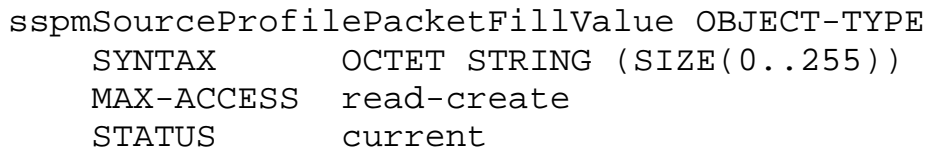




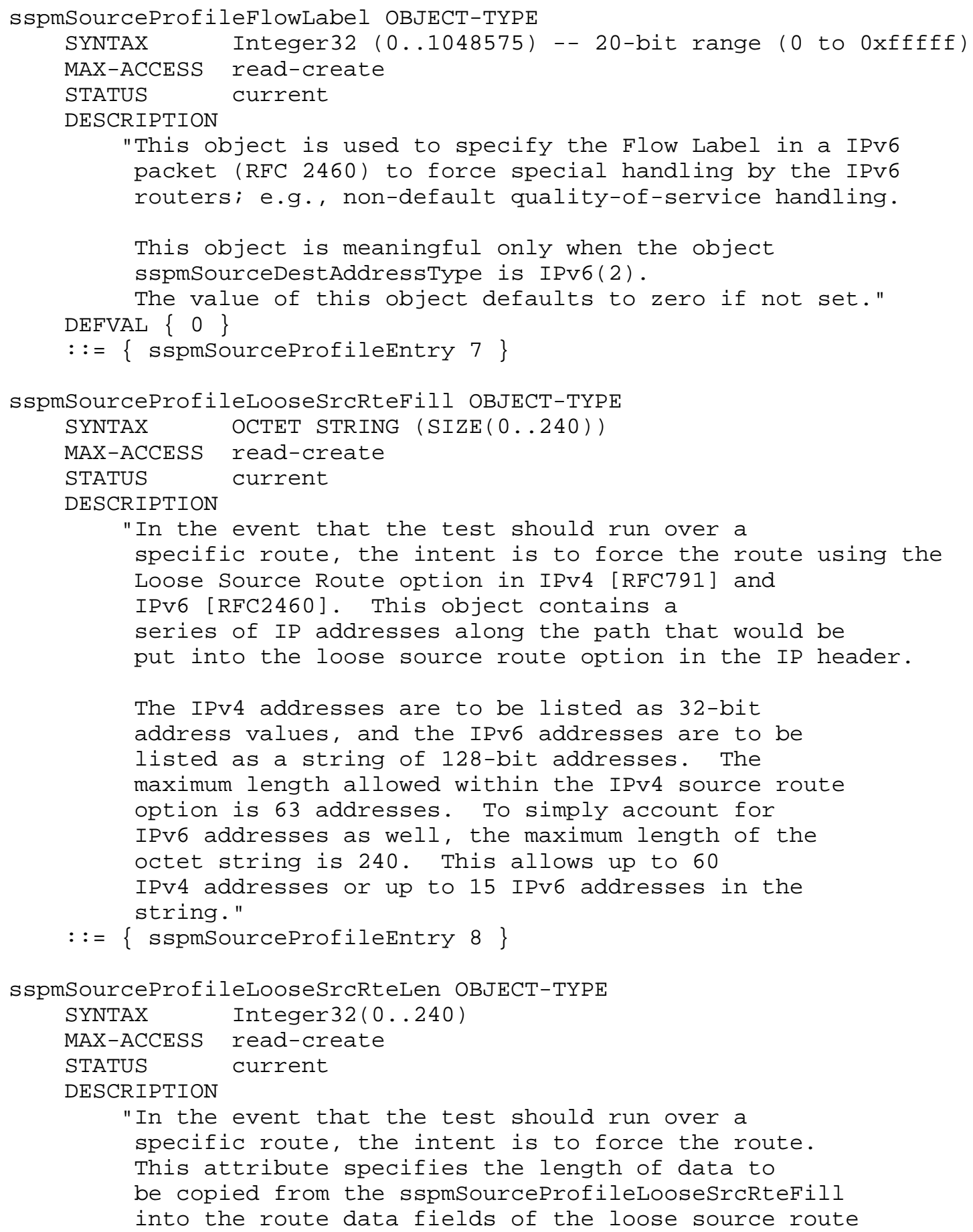




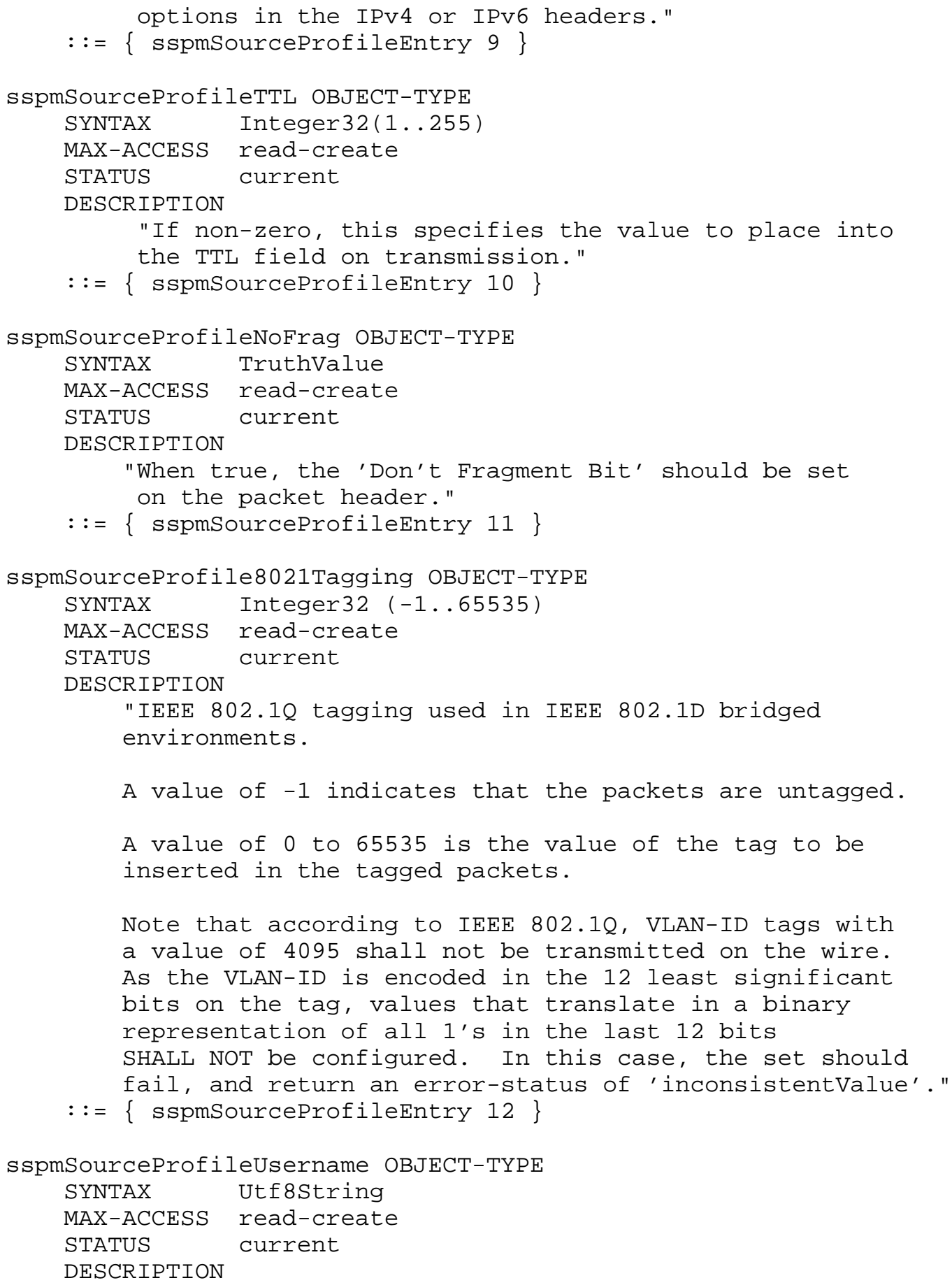




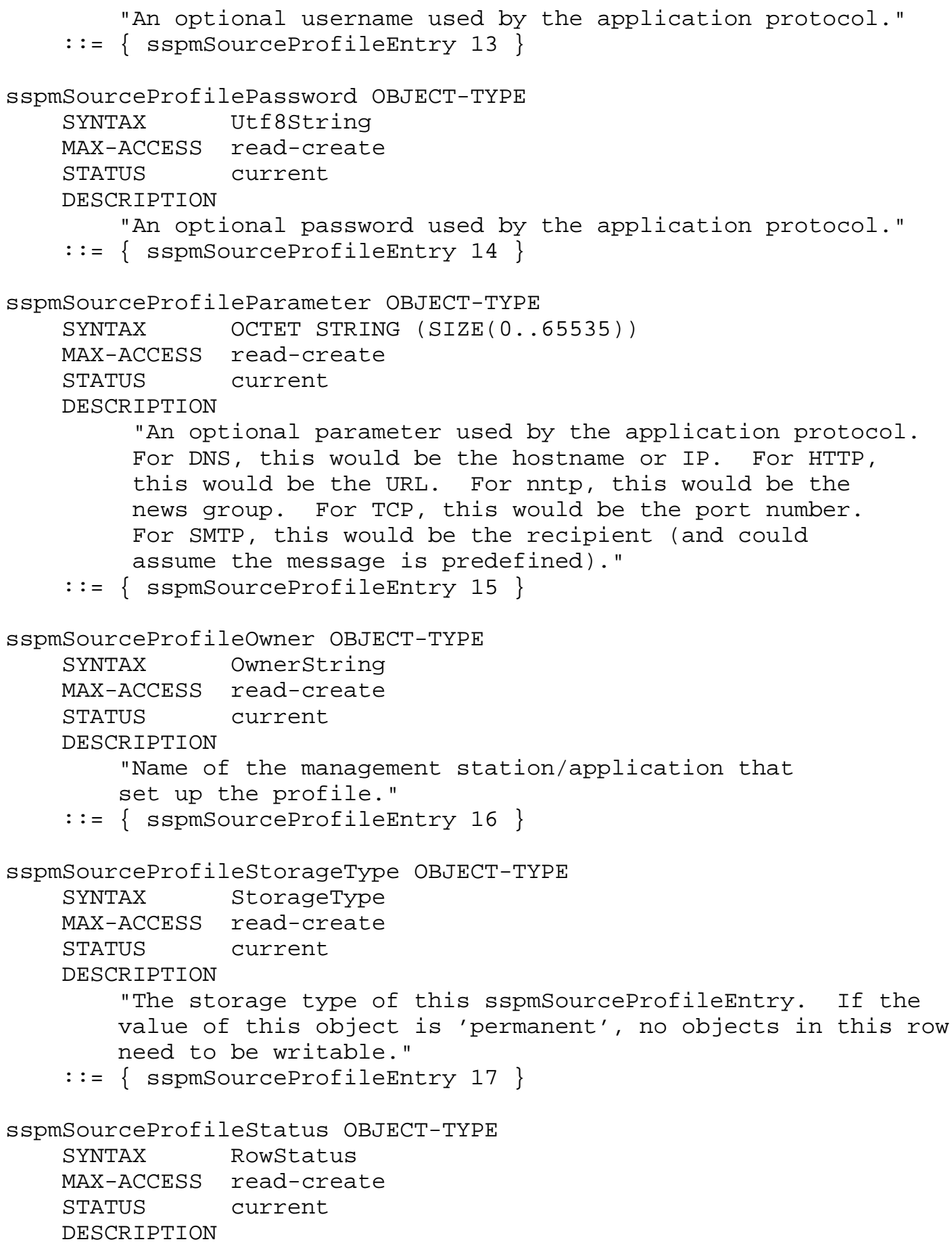




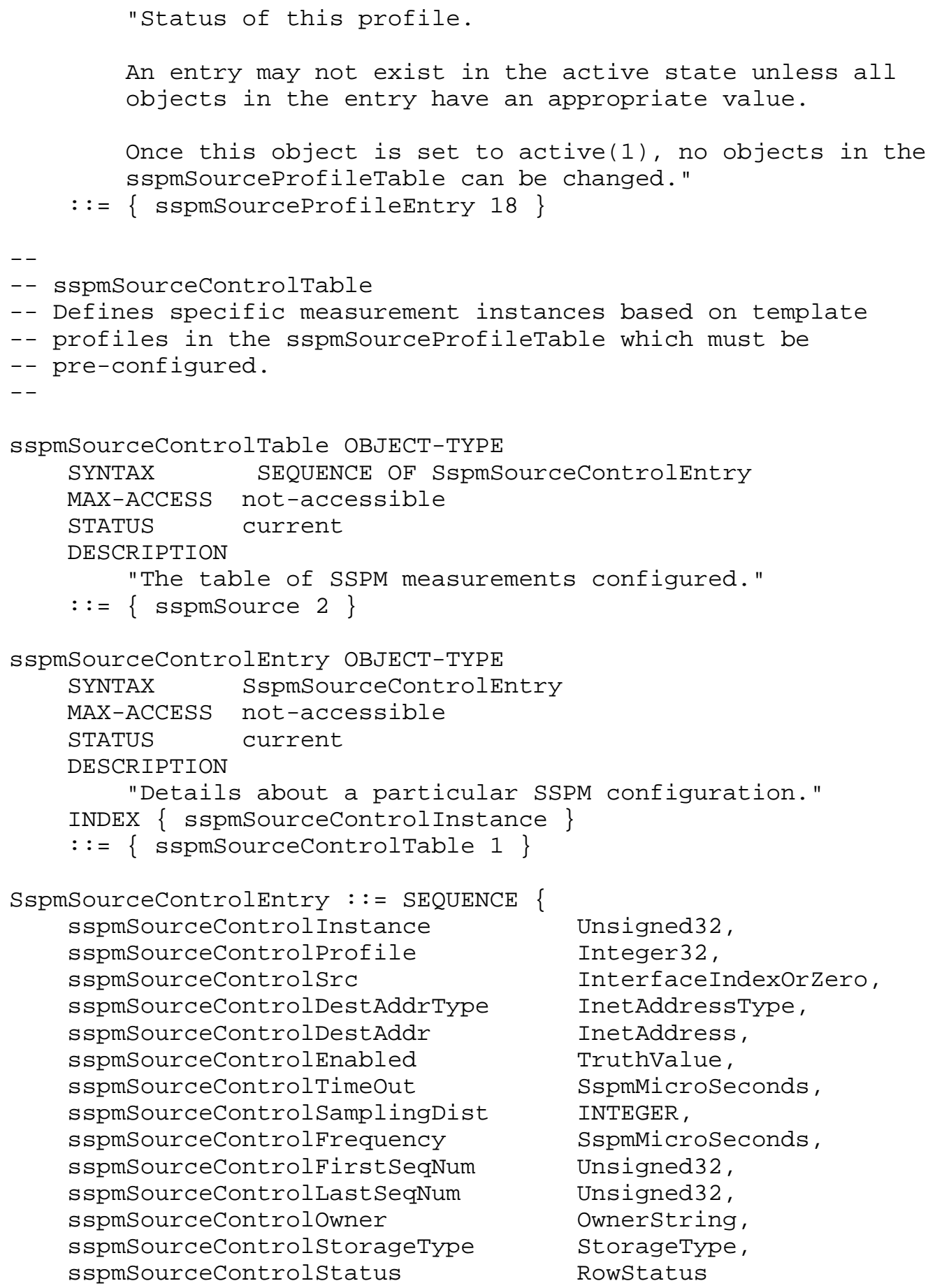

Kalbfleisch, et al. Standards Track 
\}

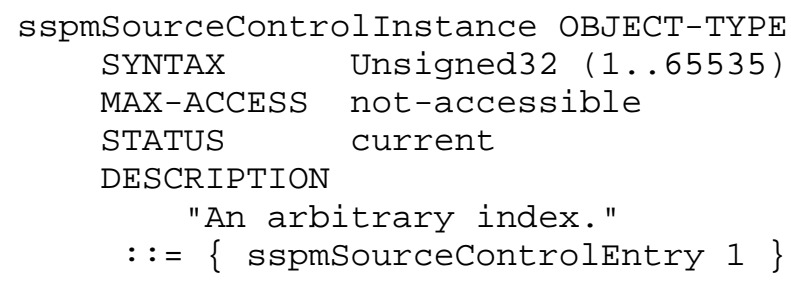




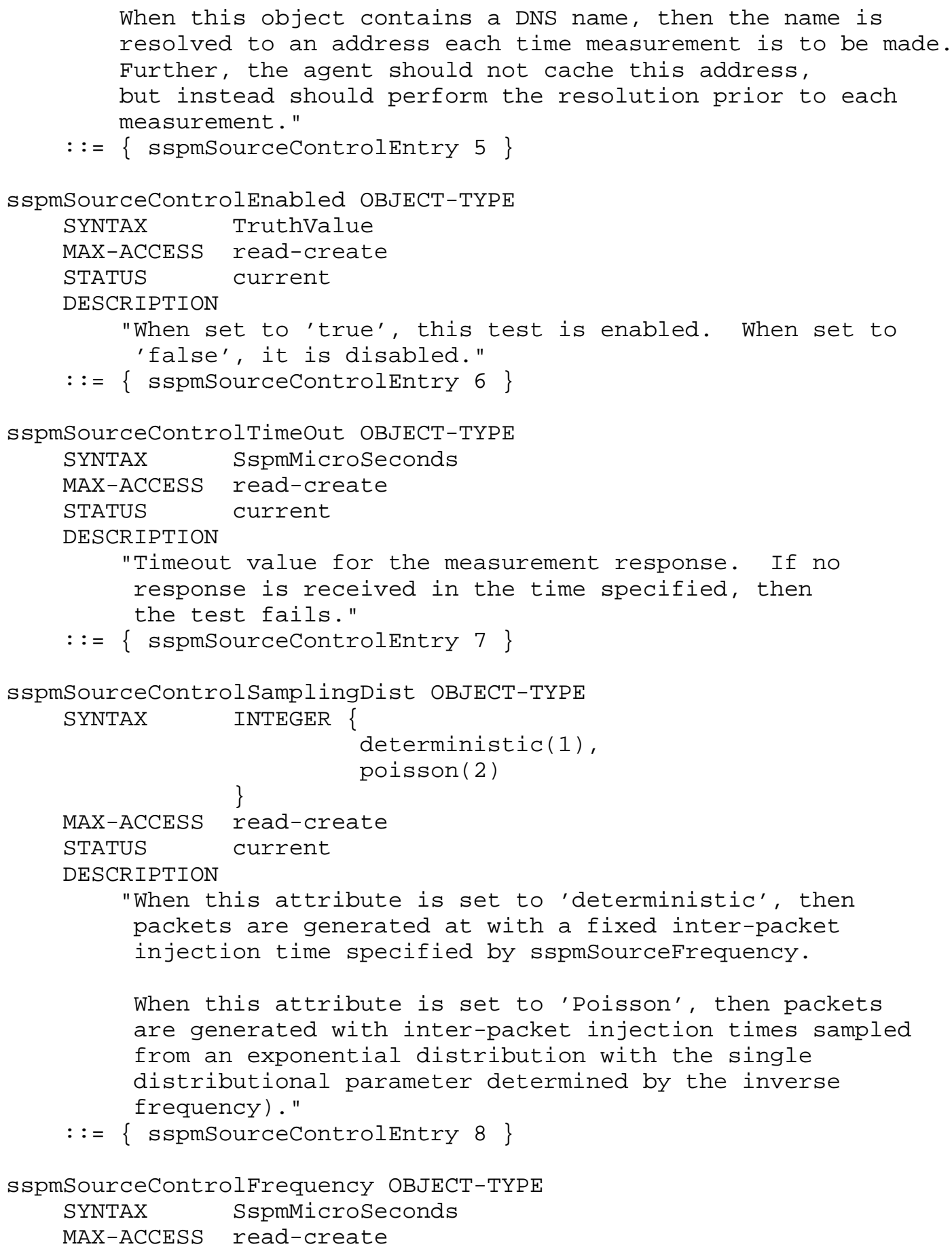




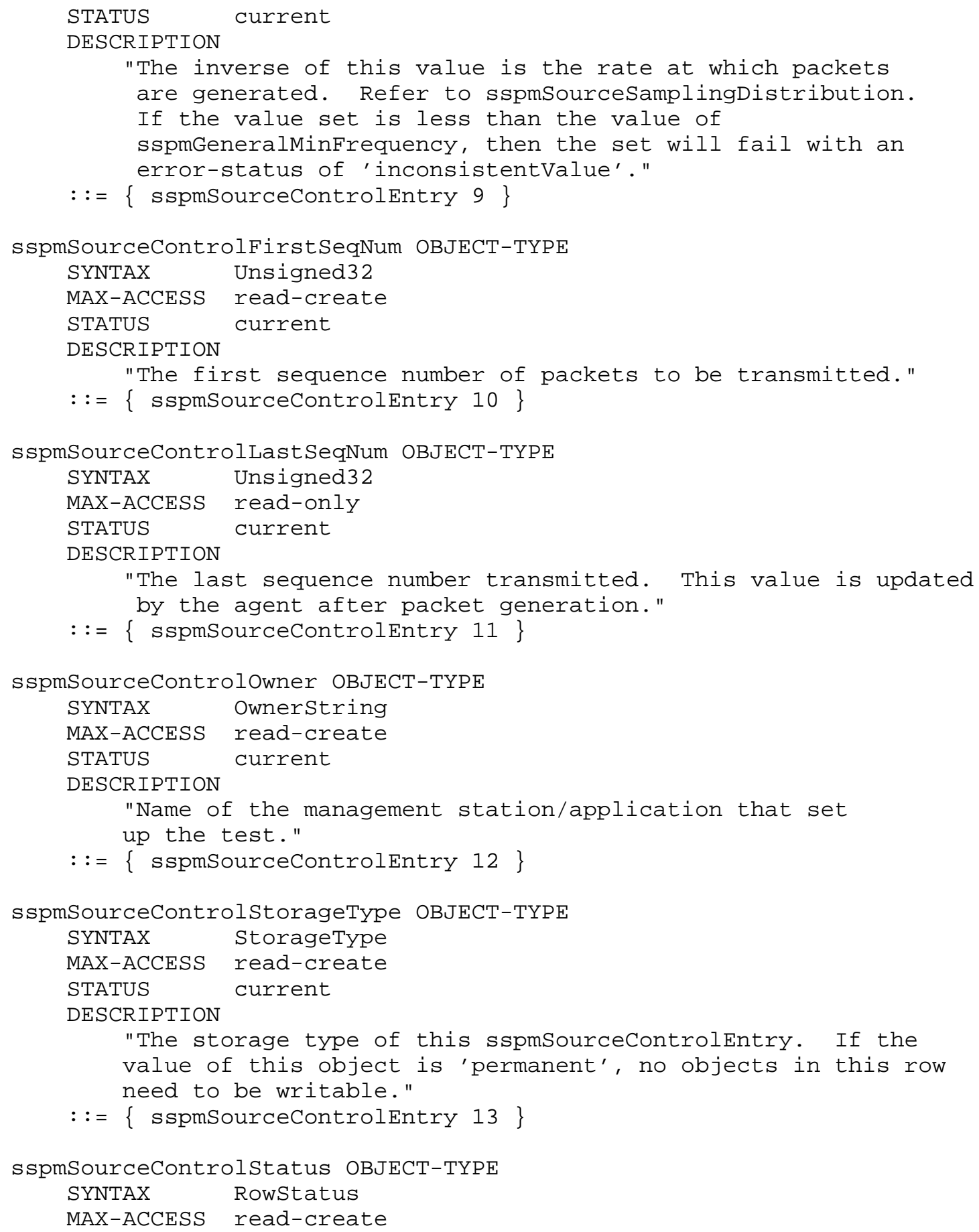




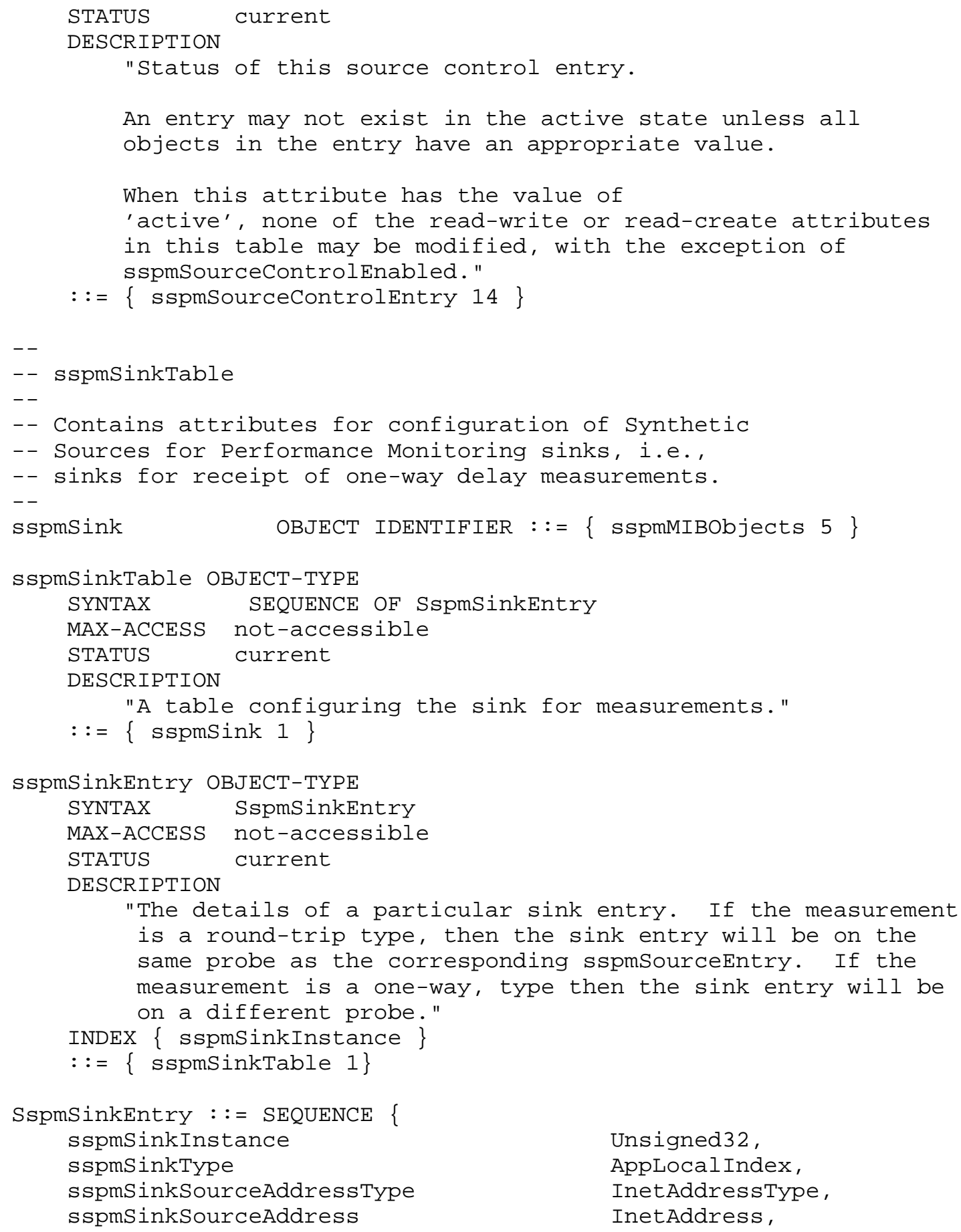

Kalbfleisch, et al. Standards Track [Page 25] 


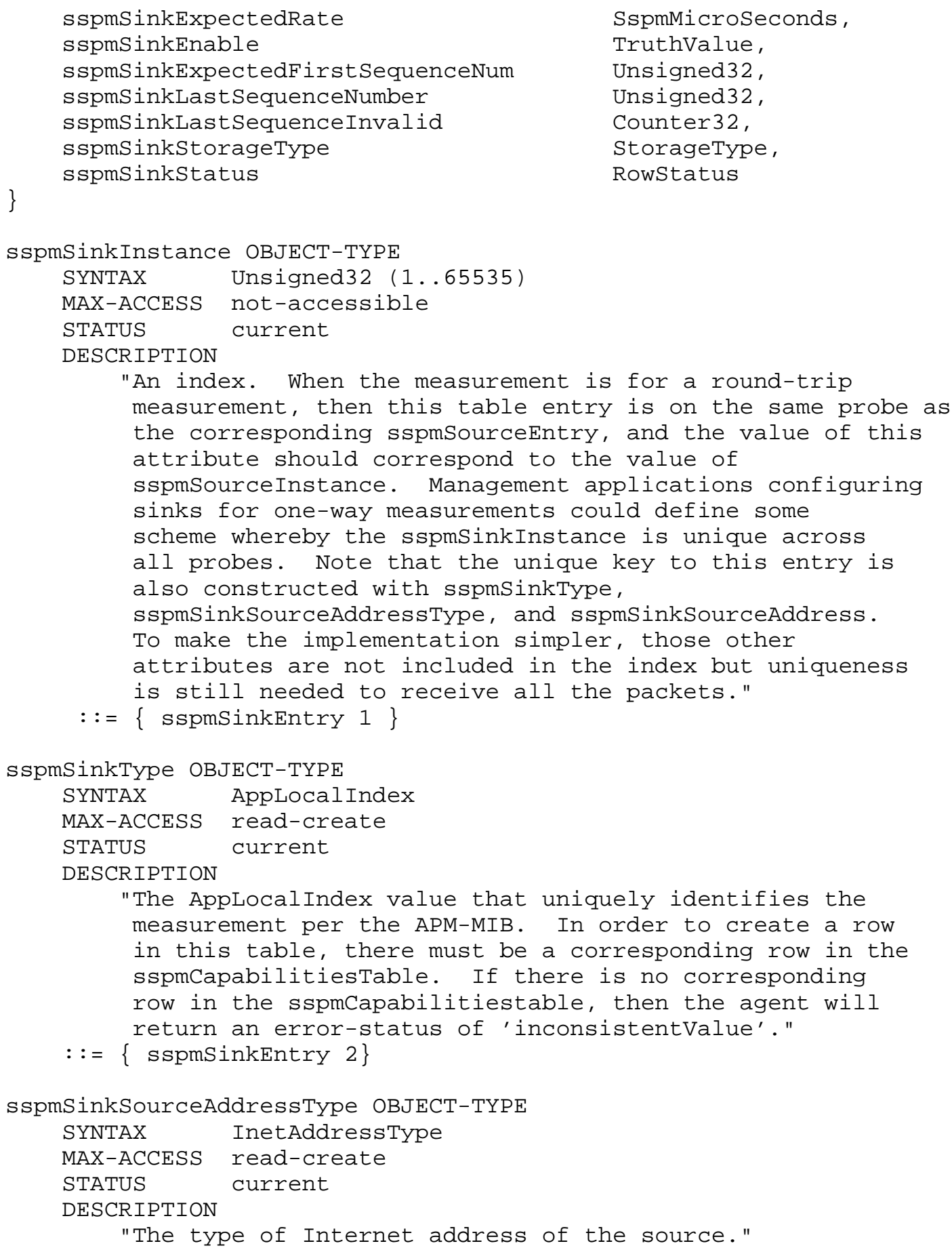




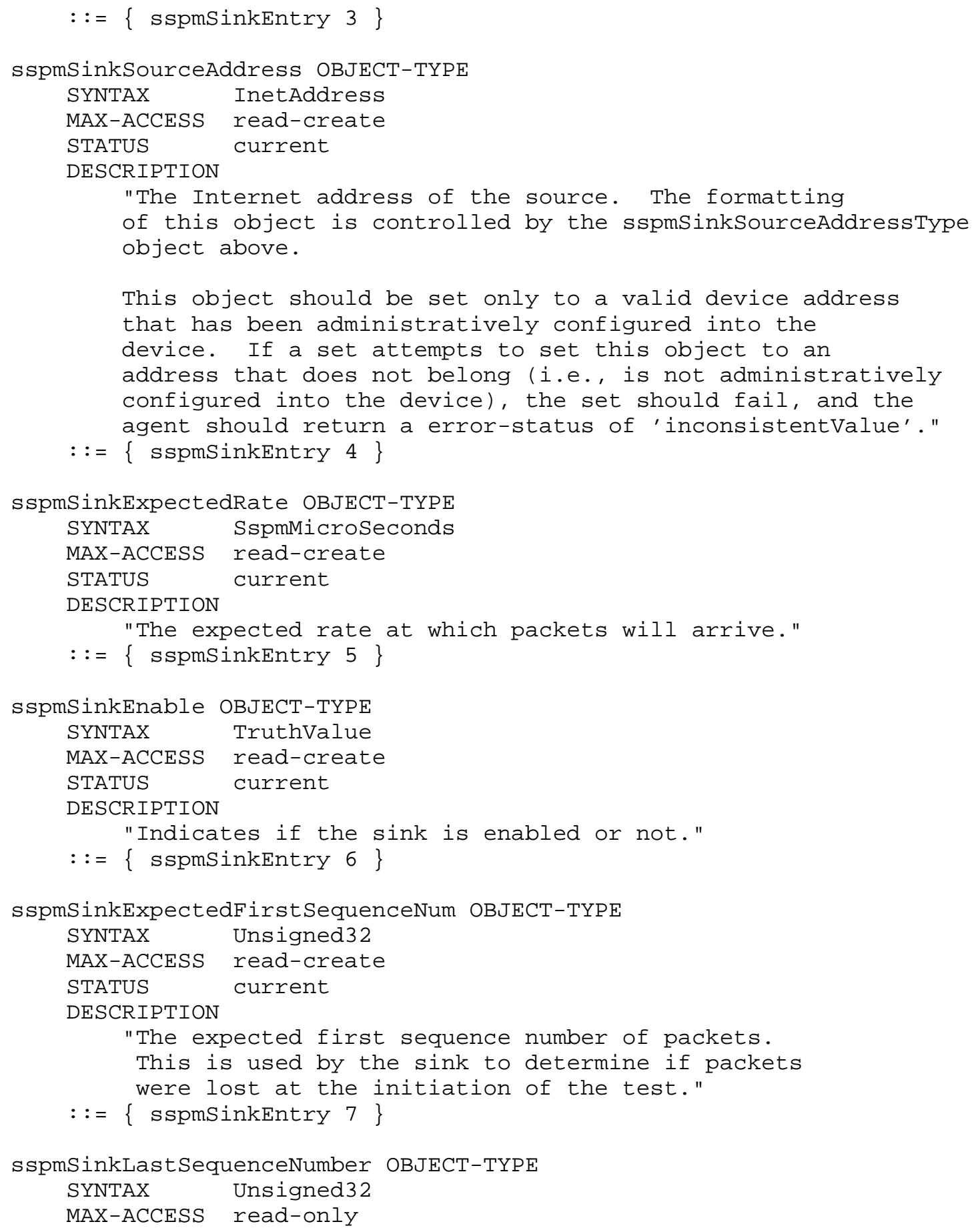




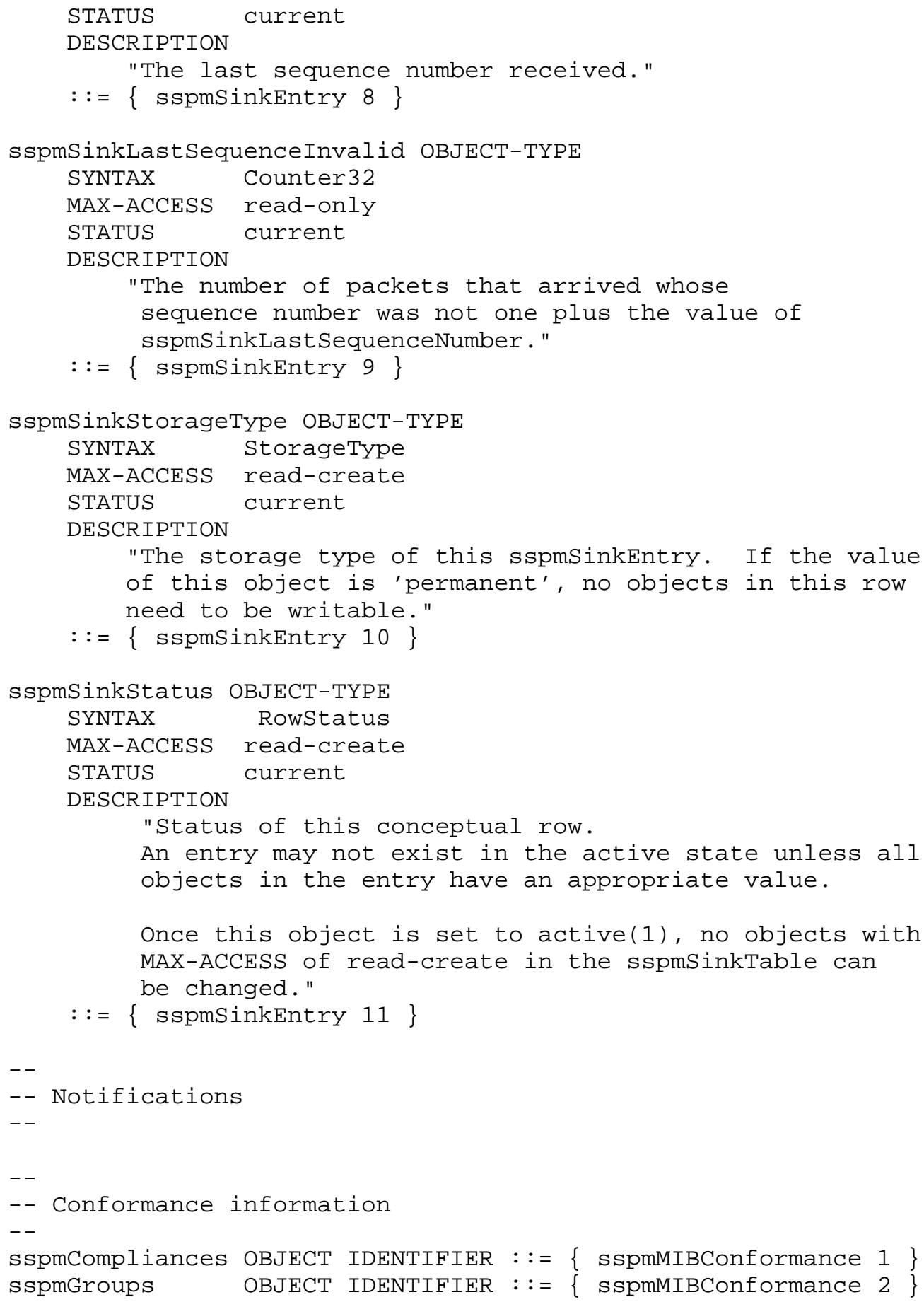

Kalbfleisch, et al. Standards Track [Page 28] 


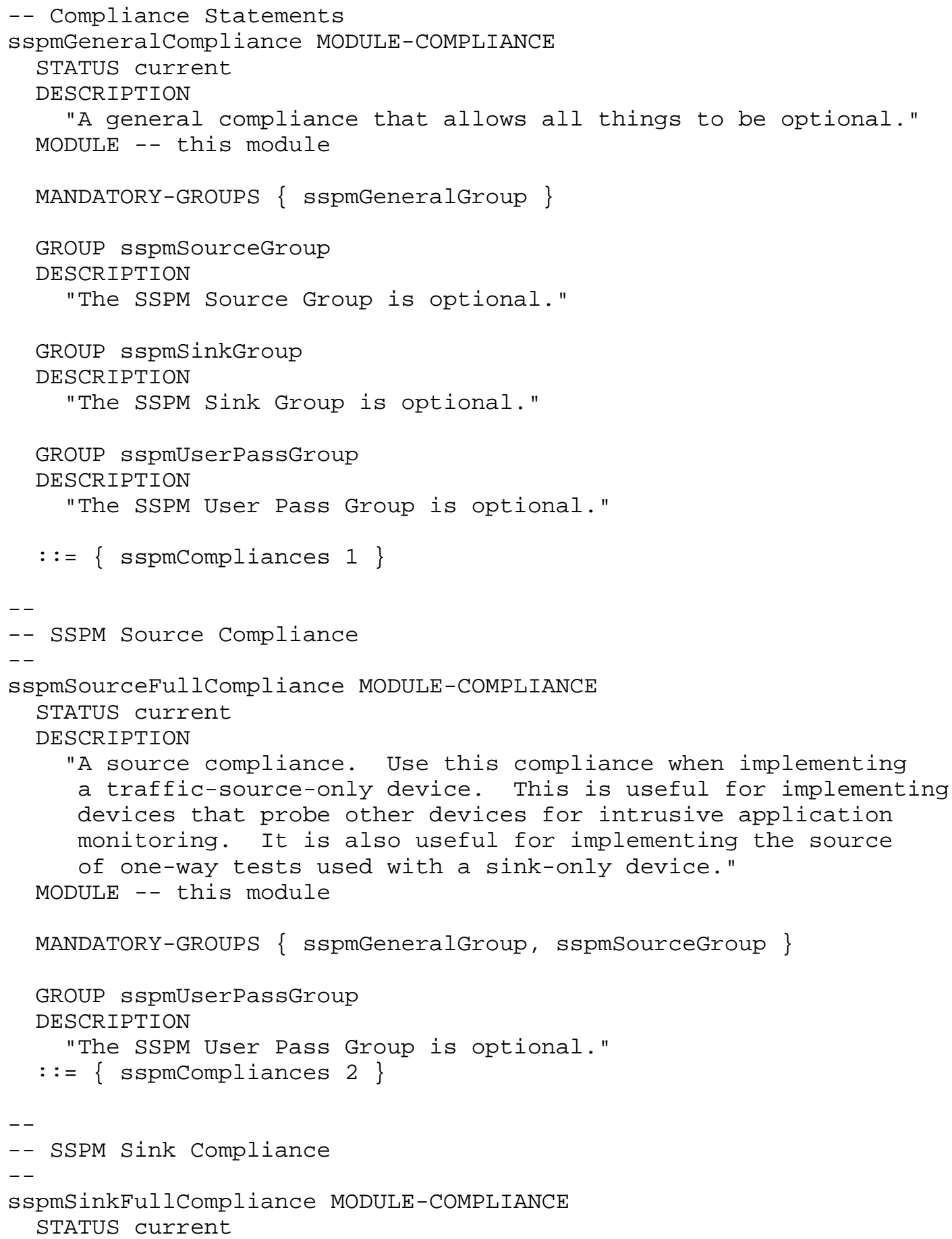




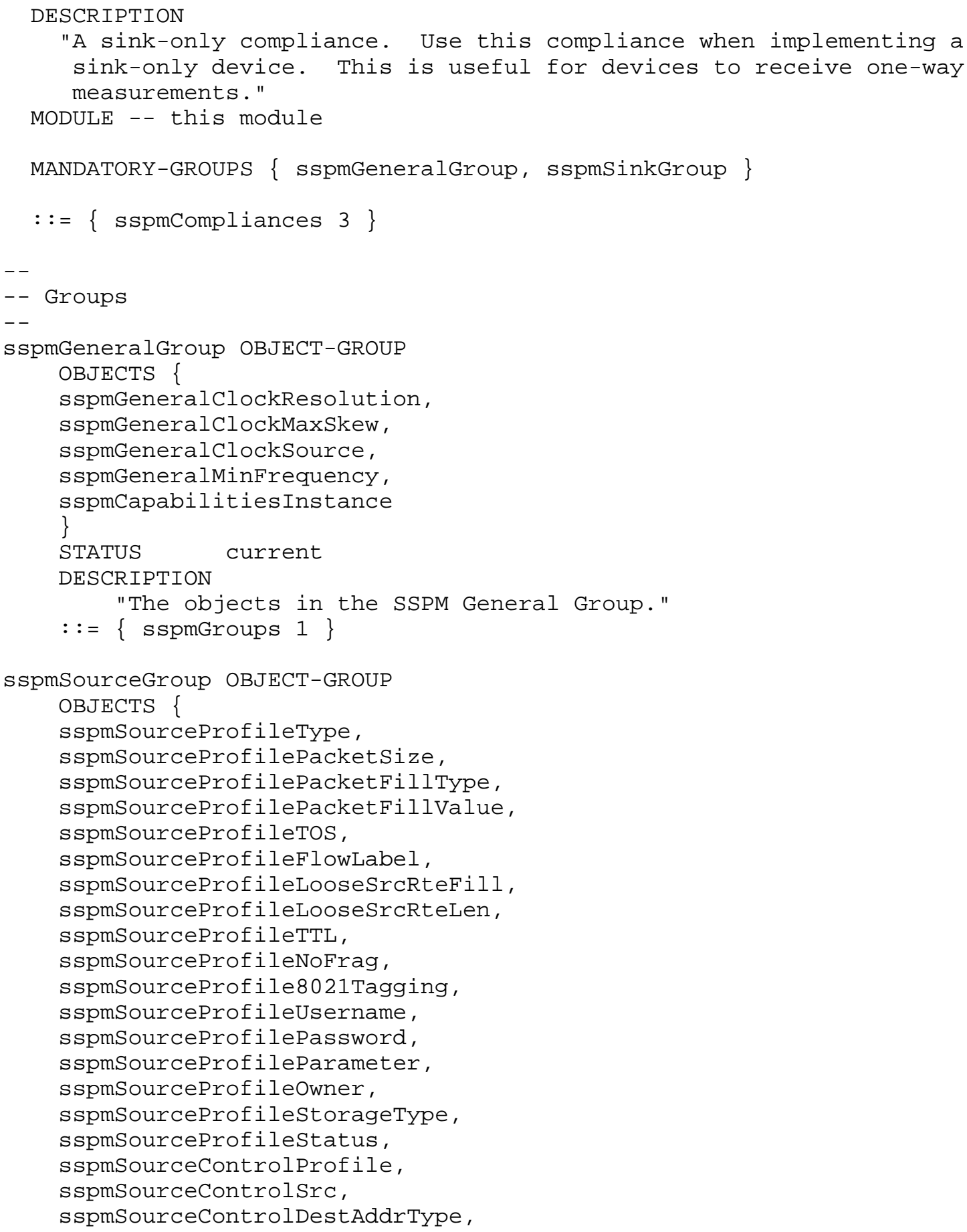

Kalbfleisch, et al. 


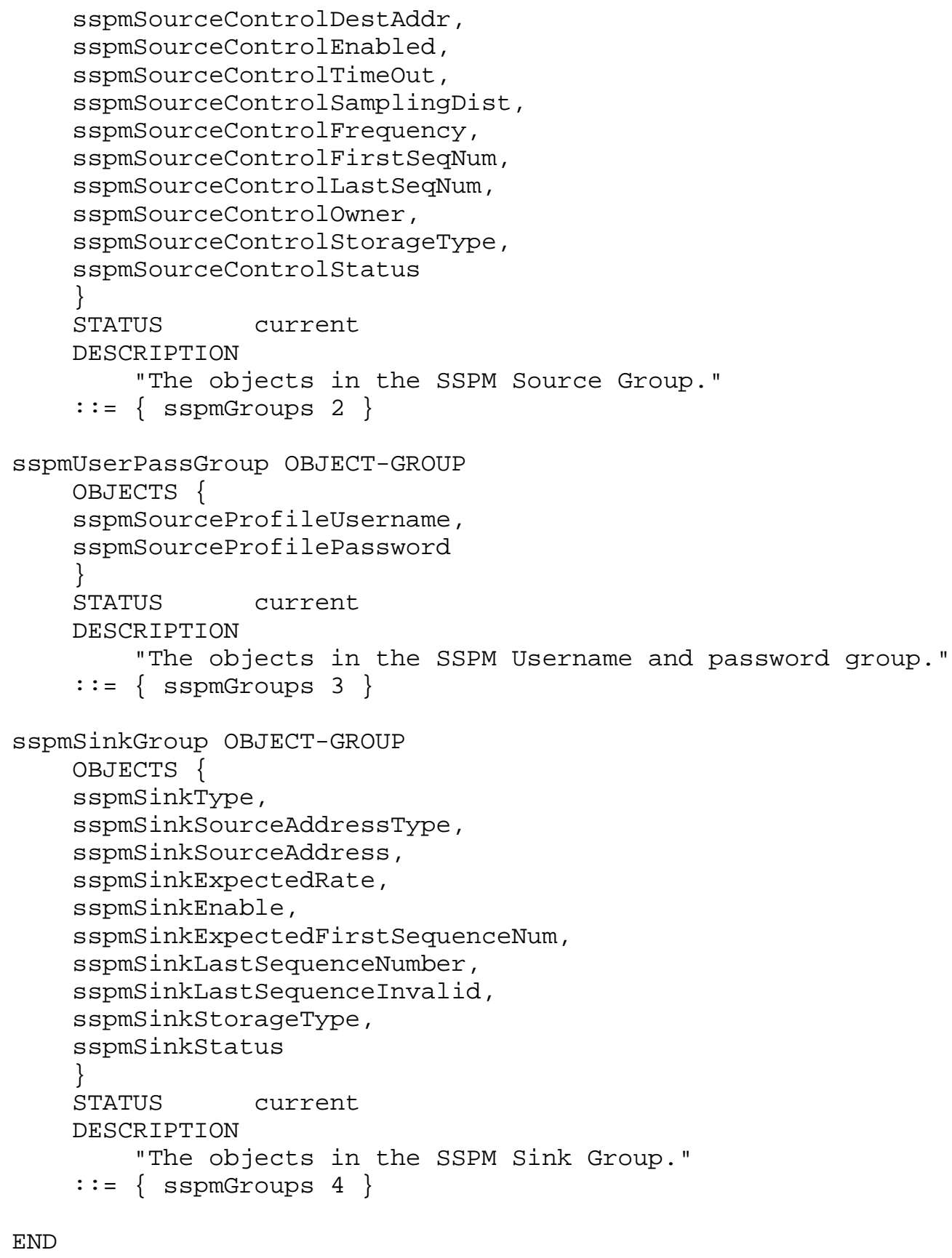

END 


\section{Security Considerations}

This MIB module defines objects that allow packets to be injected into the network for the purpose of measuring some performance characteristics. As such, the MIB module may contain sensitive network and application data; e.g., user IDs and passwords. Further, if security is compromised, this MIB module could provide a source for denial-of-service, and potential other, attacks. These issues will be addressed within this section.

There are a number of management objects defined in this MIB module that have a MAX-ACCESS clause of read-write and/or read-create. Such objects may be considered sensitive or vulnerable in some network environments. The support for SET operations in a non-secure environment without proper protection can have a negative effect on network operations. These are the tables and objects and their sensitivity/vulnerability:

+ The sspmSourceprofiletable contains objects that configure linklevel, IP, and application-level data used within test suites. These objects with a MAX-ACCESS clause of read-write and/or read- create are:

o sspmSourcePacketSize - configures the overall size of the test packets,

- sspmSourceprofiletos - sets the TOS field in the IPv4 and IPv6 headers,

- sspmSourceProfileLooseSrcRteFill and sspmSourceProfileLooseSrcRteLen - give a list of IPv4 or IPv6 addresses for the loose source route options in the IP headers,

o sspmSourceProfileflowLabel - sets the Flow Label in the IPv6 header,

o sspmSourceProfiletTL - sets the TTL field in the packet headers,

o sspmSourceProfileNoFrag - sets the No Fragment bit in the packet headers,

- sspmSourceprofile8021Tagging - sets the Tag field in the 802.1 headers, and 
- sspmSourceProfileUsername and sspmSourceProfilePassword these hold the ID and passwords specific to an application test profile.,

+ The sspmSourceControlTable contains objects that configure IP and application-level data used within a given test. These objects with a MAX-ACCESS clause of read-write and/or readcreate are:

o sspmSourceControlSrc - controls the source IP address used on the test packets,

- sspmSourceControlDestAddr - holds the destination address for the specific test packet,

o sspmSourceControlTimeout, sspmSourceControlsamplingDist, and sspmSourceControlfrequency - control the nature and frequency of the test packet injection onto the network, and

- sspmSourceControlfirstSeqNum and sspmSourceControllast SeqNum - set the first and last sequence numbers for the specific test.

+ The sspmSinkTable contains objects that configure the recipient of the test packets. As such, the objects in this table have no security issues related to them.

Some attributes configure username and password information for some application-level protocols as indicated above. Access to these attributes may provide unauthorized use of resources. These attributes are: sspmSourceProfileUsername and sspmSourceprofilePassword.

Some attributes configure the size and rate of traffic flows for the purpose of performance measurements. Access to these attributes may exacerbate the use of this MIB module in denial-of-service attacks. It is possible to define a maximum packet rate on the device and to indicate this rate through the sspmSourceFrequency object. This object reflects the maximum acceptable packet rate that a device supporting this MIB module is willing to generate. This places a bound on setting the test packet rate through the sspmSourceControlfrequency object. Other objects that control aspects of the test packets related to packet size and rate are sspmSourceControlTimeOut, sspmSourceControlsamplingDist and sspmSourceControlfrequency. 
The objects sspmSourceControlSrc, sspmSourceControldestAddr, sspmSourceControlLooseSrcRteFill, and sspmSourceControlLooseSrcRteLen control the setting of the source and destination addresses on the packet headers and the routing of the packets. The device should not allow the setting of source addresses on the test packets other than those that are administratively configured onto the device. This is controlled by using the syntax InterfaceIndexorzero for the control of the source address through the sspmSourceControlsrc object.

It is thus important to control even GET access to these objects and possibly to even encrypt the values of these object when sending them over the network via SNMP. Not all versions of SNMP provide features for such a secure environment.

SNMP versions prior to SNMPV3 did not include adequate security. Even if the network itself is secure (for example by using IPSec), even then, there is no control as to who on the secure network is allowed to access and GET/SET (read/change/create/delete) the objects in this MIB module.

It is RECOMMENDED that implementers consider the security features as provided by the SNMPV3 framework (see [RFC3410], section 8), including full support for the SNMPv3 cryptographic mechanisms (for authentication and privacy).

Further, deployment of SNMP versions prior to SNMPv3 is NOT RECOMMENDED. Instead, it is RECOMMENDED to deploy SNMPv3 and to enable cryptographic security. It is then a customer/operator responsibility to ensure that the SNMP entity giving access to an instance of this MIB module is properly configured to give access to the objects only to those principals (users) that have legitimate rights to indeed GET or SET (change/create/delete) them.

\section{Acknowledgements}

This document was produced by the IETF Remote Network Monitoring Working Group. The editors gratefully acknowledge the comments of the following individuals: Andy Bierman, Lester D'Souza, Jim McQuaid, and Steven Waldbusser.

10. Normative References

[RFC791] Postel, J., "Internet Protocol", STD 5, RFC 791, September 1981 .

[RFC1305] Mills, D., "Network Time Protocol (Version 3) Specification, Implementation and Analysis", RFC 1305, March 1992 . 
[RFC2119] Bradner, S., "Key words for use in RFCs to Indicate Requirement Levels", BCP 14, RFC 2119, March 1997.

[RFC2287] Krupczak, C. and J. Saperia, "Definitions of System-Level Managed Objects for Applications", RFC 2287, February 1998 .

[RFC2460] Deering, S. and R. Hinden, "Internet Protocol, Version 6 (IPv6) Specification", RFC 2460, December 1998.

[RFC2578] McCloghrie, K., Perkins, D., Schoenwaelder, J., Case, J., Rose, M., and S. Waldbusser, "Structure of Management Information Version 2 (SMIv2)", STD 58, RFC 2578, April 1999.

[RFC2579] MCCloghrie, K., Perkins, D., Schoenwaelder, J., Case, J., Rose, M., and S. Waldbusser, "Textual Conventions for SMIV2", STD 58, RFC 2579, April 1999.

[RFC2580] McCloghrie, K., Perkins, D., Schoenwaelder, J., Case, J., Rose, M., and S. Waldbusser, "Conformance Statements for SMIV2", STD 58, RFC 2580, April 1999.

[RFC2680] Almes, G., Kalidindi, S., and M. Zekauskas, "A One-Way Packet Loss Metric for IPPM" RFC 2680, september 1999.

[RFC2863] MCCloghrie, K. and F. Kastenholz, "The Interfaces Group MIB", RFC 2863, June 2000 .

[RFC3291] Daniele, M., Haberman, B., Routhier, S., and J. Schoenwaelder, "Textual Conventions for Internet Network Addresses ", RFC 3291, May 2002 .

[RFC3393] Demichelis, C. and P. Chimento, "IP Packet Delay Variation Metric for IP Performance Metrics (IPPM)", RFC 3393, November 2002 .

[RFC3432] Raisanen, V., Grotefeld, G., and A. Morton, "Network Performance Measurement with Periodic Streams", RFC 3432, November 2002 .

[RFC3577] Waldbusser, S., Cole, R.G., Kalbfleisch, C., and D. Romascanu, "Introduction to the Remote Monitoring (RMON) Family of MIB Modules", RFC 3577, August 2003.

[RFC3729] Waldbusser, S., "Application Performance Measurement MIB", RFC 3729, March 2004. 
[RFC4150] Dietz, R. and R. Cole, "Transport Performance Metrics MIB", RFC 4150, August 2005 .

11. Informative References

[RFC1272] Mills, C., Hirsch, G., and G. Ruth, "Internet Accounting Background", RFC 1272, November 1991.

[RFC2021] Waldbusser, S., "Remote Network Monitoring Management Information Base Version 2 using SMIv2", RFC 2021, January 1997 .

[RFC2722] Browlee, N., Mills, C., and G. Ruth, "Traffic Flow Measurement: Architecture", RFC 2722, October 1999.

[RFC2720] Brownlee, N. "Traffic Flow Measurement: Meter MIB", RFC 2720, October 1999.

[RFC2330] Paxson, V., Almes, G., Mahdavi, J., and M. Mathis, "Framework for IP Performance Metrics", RFC 2330, May 1998 .

[RFC2564] Kalbfleisch, C., Krupczak, C., Presuhn, R., and J. Saperia, "Application Management MIB", RFC 2564, May 1999 .

[RFC2594] Hazewinkel, H., Kalbfleisch, C., and J. Schoenwaelder, "Definitions of Managed Objects for WWW Services", RFC 2594, May 1999.

[RFC3165] Levi, D. and J. Schoenwaelder, "Definitions of Managed Objects for the Delegation of Management Scripts", RFC 3165, August 2001 .

[RFC2678] Mahdavi, J. and V. Paxson, "IPPM metrics for Measuring Connectivity", RFC 2678, September 1999.

[RFC2679] Almes, G., Kalidindi, S., and M. Zekauskas, "A One-way Delay Metric for IPPM", RFC 2679, September 1999.

[RFC2681] Almes, G., Kalidindi, S., and M. Zekauskas, "A Round-Trip Delay Metric for IPPM", RFC 2681, September 1999.

[RFC2819] Waldbusser, S., "Remote Network Monitoring Management Information Base", STD 59, RFC 2819, February 1995. 
[RFC2925] White, K., "Definitions of Managed Objects for Remote Ping, Traceroute, and Lookup Operations", RFC 2925, September 2000 .

[RFC2982] Kavasseri, R., "Distributed Management Expression MIB", RFC 2982, October 2000 .

[RFC3410] Case, J., Mundy, R., Partain, D., and B. Stewart, "Introduction and Applicability Statements for InternetStandard Management Framework", RFC 3410, December 2002.

[RFC3512] MacFaden, M., Partain, D., Saperia, J., and W. Tackabury, "Configuring Networks and Devices with Simple Network Management Protocol (SNMP)", RFC 3512, April 2003.

[EBT] Mathis, M. and M. Allman, "Empirical Bulk Transfer Capacity", Work in Progress, October 1999.

[ODP] Shalunov, S., Teitelbaum, B., and M. Zekauskas, "A OneWay Delay Protocol for IP Performance Measurements", Work in Progress, December 2000.

[RFC4011] Waldbusser, S., Saperia, J., and T. Hongal, "Policy Based Management MIB", RFC 4011, March 2005.

Mathis, M., "TReno Bulk transfer Capacity", Work in Progress, February 1999. 


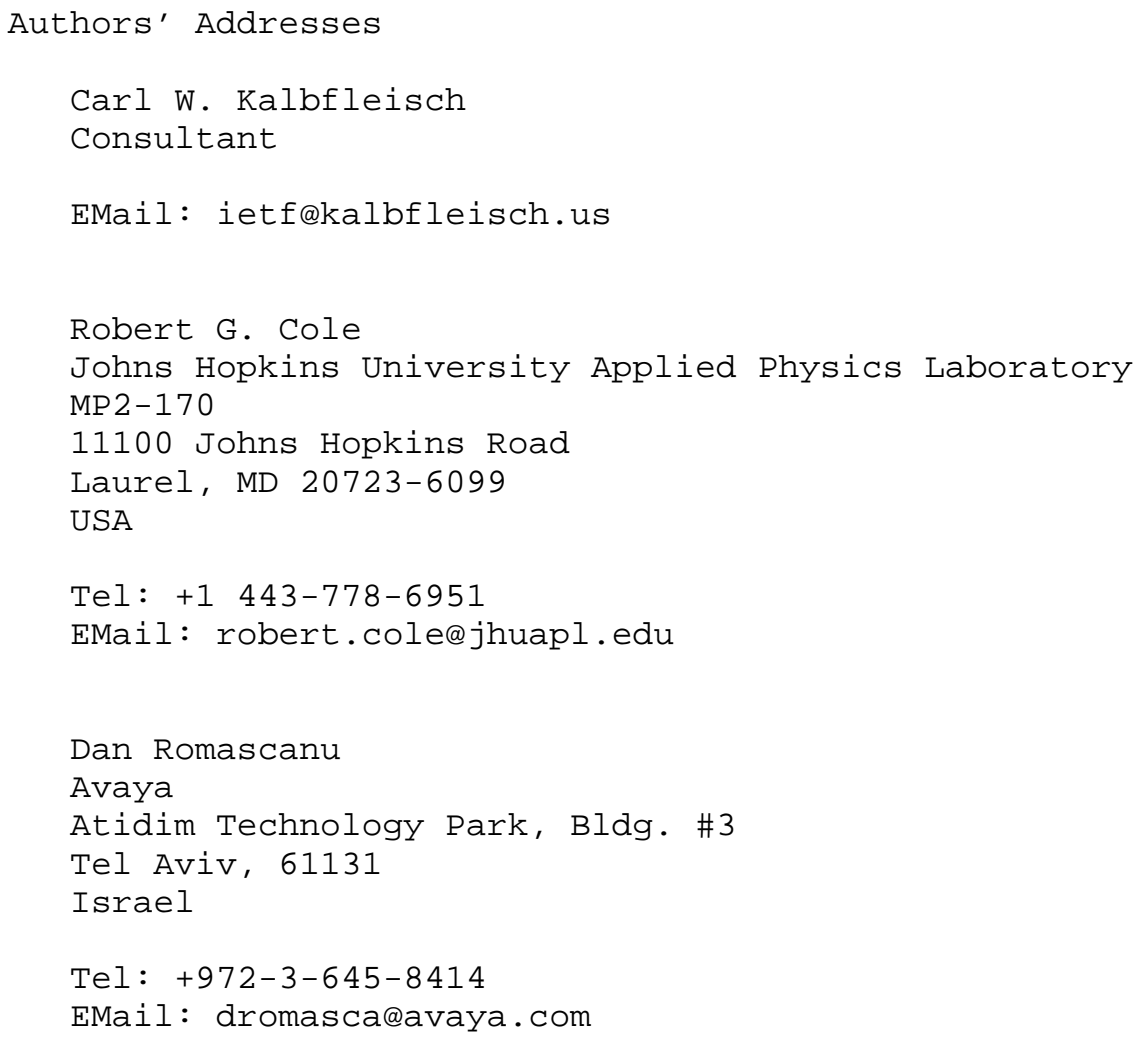


Full Copyright statement

Copyright (C) The Internet Society (2005).

This document is subject to the rights, licenses and restrictions contained in BCP 78, and except as set forth therein, the authors retain all their rights.

This document and the information contained herein are provided on an "AS IS" basis and THE CONTRIBUTOR, THE ORGANIZATION HE/SHE REPRESENTS OR IS SPONSORED BY (IF ANY), THE INTERNET SOCIETY AND THE INTERNET ENGINEERING TASK FORCE DISCLAIM ALL WARRANTIES, EXPRESS OR IMPLIED, INCLUDING BUT NOT LIMITED TO ANY WARRANTY THAT THE USE OF THE INFORMATION HEREIN WILL NOT INFRINGE ANY RIGHTS OR ANY IMPLIED WARRANTIES OF MERCHANTABILITY OR FITNESS FOR A PARTICULAR PURPOSE.

Intellectual Property

The IETF takes no position regarding the validity or scope of any Intellectual property Rights or other rights that might be claimed to pertain to the implementation or use of the technology described in this document or the extent to which any license under such rights might or might not be available; nor does it represent that it has made any independent effort to identify any such rights. Information on the procedures with respect to rights in RFC documents can be found in BCP 78 and BCP 79 .

Copies of IPR disclosures made to the IETF Secretariat and any assurances of licenses to be made available, or the result of an attempt made to obtain a general license or permission for the use of such proprietary rights by implementers or users of this specification can be obtained from the IETF on-line IPR repository at http://www.ietf.org/ipr.

The IETF invites any interested party to bring to its attention any copyrights, patents or patent applications, or other proprietary rights that may cover technology that may be required to implement this standard. Please address the information to the IETF at ietfipreietf.org.

Acknowledgement

Funding for the RFC Editor function is currently provided by the Internet Society. 